Published in final edited form as:

Nat Immunol. 2018 March ; 19(3): 279-290. doi:10.1038/s41590-018-0046-x.

\title{
Hoxb5 reprograms B cells into functional T lymphocytes
}

Mengyun Zhang ${ }^{1,2, \dagger}$, Yong Dong ${ }^{1,2, \dagger}$, Fangxiao $\mathrm{Hu}^{1, \dagger}$, Dan Yang ${ }^{1}$, Qianhao Zhao ${ }^{1}$, Cui Lv ${ }^{1}$, Ying Wang ${ }^{1,2}$, Chengxiang Xia ${ }^{1,2}$, Qitong Weng ${ }^{1,2}$, Xiaofei Liu ${ }^{1}$, Chen Li ${ }^{3}$, Peiqing Zhou ${ }^{1,2}$, Tongjie Wang ${ }^{1}$, Yuxian Guan ${ }^{1}$, Rongqun Guo ${ }^{1,2}$, Lijuan Liu${ }^{1}$, Yang Geng ${ }^{1}$, Hongling Wu ${ }^{1}$, Juan $\mathrm{Du}^{1}$, Zheng Hu${ }^{4}$, Sheng $\mathrm{Xu}^{5}$, Jiekai Chen ${ }^{1,2}$, Aibin $\mathrm{He}^{3}$, Bing Liu ${ }^{6}$, Demin Wang ${ }^{7,8}$, Yong-Guang Yang ${ }^{4,9}$, and Jinyong Wang ${ }^{1,2, *}$

${ }^{1}$ CAS Key Laboratory of Regenerative Biology, Guangzhou Institutes of Biomedicine and Health, Guangzhou, China.

2University of Chinese Academy of Sciences, Beijing, 100049, China.

${ }^{3}$ Institute of Molecular Medicine, Beijing Key Laboratory of Cardiometabolic Molecular Medicine, Peking University, Beijing 100871, China.

${ }^{4}$ The First Hospital and Institute of Immunology, Jilin University, Changchun, China.

${ }^{5}$ National key laboratory of medical immunology \&institute of immunology, second military medical university, Shanghai, China.

${ }^{6}$ State Key Laboratory of Proteomics, Translational Medicine Center of Stem cells, 307-Ivy Translational Medicine Center, Laboratory of Oncology, Affiliated Hospital, Academy of Military Medical Sciences, Beijing, China.

${ }^{7}$ Biomedical Research Center of South China, College of Life Sciences, Fujian Normal University, Fuzhou, Fujian 350117, China.

${ }^{8}$ Blood Research Institute, Blood Center of Wisconsin, Milwaukee, WI, USA.

${ }^{9}$ Columbia Center for Translational Immunology, Columbia University College of Physicians and Surgeons, New York, USA.

\section{Abstract}

Deletion of B cell master regulators reprogrammed B cells into $\mathrm{T}$ cells that were either functional defects or tumorigenic potential. Here we show that Hoxb5, which is expressed in uncommitted

*Correspondence to J.Y.W. (wang_jinyong@ gibh.ac.cn).

$\dagger$ Equal contributions.

AUTHOR CONTRIBUTIONS

M.Z., Y.D., and F.H. performed the core experiments and contributed equally to this work. D.Y., Q.Z., C.Lv., Y.W., C.X., Q.W., X.L., and C.Li. performed multiple experiments. P.Z., T.W., Y.G., R.G., L.L., Y.G., and H.W. performed certain in vitro experiments. B.L., D.W., and J.W. wrote the manuscript. J.D., Z.H., S.X., J.C., A.H., B.L., D.W., Y-G.Y., and J.W. discussed the data and edited the manuscript. J.W. designed the project and final approval of the manuscript.

COMPETING FINANCIAL INTERESTS

The authors declare no competing interests as defined by Springer Nature, or other interests that might be perceived to influence the results and/or discussion reported in this paper.

A life Science Reporting Summary for this paper is available.

Data availability. The data that support the findings of this study are available from the corresponding author upon request. All RNASeq and CHIP-Seq data are in the GEO database with accession code GSE105057. 
hematopoietic progenitors but absent in committed B and T lineages, could reprogram pro-pre-B cells into functional early $\mathrm{T}$ cell progenitors. The reprogramming started in bone marrow and completed in thymus, giving rise to $\mathrm{T}$ lymphocytes with transcriptomes, hierarchical differentiation, tissue distribution and immune functions closely resembling their natural counterparts. Hoxb5 repressed B cell master genes, activated T cell regulators and regulated crucial chromatin modifiers in pro-pre-B cells, ultimately driving B to $\mathrm{T}$ cell fate conversion. Our results provide a de novo paradigm for generating normal and functional $\mathrm{T}$ cells through reprogramming in vivo.

Cell fate is strictly controlled during development, ensuring that each cell contributes to organismal function in a harmonious fashion. Multi- or pluripotent cells can be directed to differentiate to a specific cell type or trans-differentiate from one lineage to another by expression of particular transcription factors. Master regulators that can mediate hematopoietic lineage conversion have been identified. As such, expression of Gatal converts monocytic precursors into erythroid-megakaryocytic cells and eosinophils ${ }^{1-3}$ and Cebpa converts B cells into macrophages ${ }^{4}$; deletion of Pax 5 converts B cells into uncommitted hematopoietic progenitors ${ }^{5,6}$; expression of Gata3 converts T lymphocytes into mast cells ${ }^{7}$; expression of Cebp \pm and Spi1 converts T lymphocytes into macrophages and dendritic cells ${ }^{8}$ and deletion of $B c 111 b$ converts $\mathrm{T}$ lymphocytes into natural killer-like cells ${ }^{9}$. Attempts to convert B to T cells by silencing B lineage master genes have had limited success, in that it has not been possible to reconstitute the entire $\mathrm{T}$ lineage functionally, and in some instances, the manipulations increased cancer risk $5,6,10,11$. In aggregate, these studies indicate that hematopoietic cell fate can be manipulated genetically.

Hematopoietic stem cells (HSC) and multipotent progenitors (MPP) differentiate into various hematopoietic cell types through activation of specific gene regulatory networks 12,13. The transcription factor Hoxb5 is specifically expressed in HSC ${ }^{14}$, although the entire Hoxb gene cluster appears to be dispensable for hematopoiesis ${ }^{15}$. Here, we show that expression of Hoxb5 alone in pro-pre-B cells, followed by transplantation of the pro-preB cells into sublethally-irradiated recipient mice, produced early T cell progenitors (ETPs) in bone marrow and ultimately regenerated a full complement of functional $\mathrm{T}$ lymphocytes, whose transcriptomes, hierarchical differentiation, tissue distribution and immune functions closely resemble those of endogenous $\mathrm{T}$ lymphocytes. To our knowledge, this is the first report of a procedure for generating fully functional $\mathrm{T}$ lymphocytes in vivo by lineageconversion.

\section{RESULTS}

\section{Ectopic expression of 15 factors reprograms B cells into T cells}

First, we tested whether hematopoietic cells could be converted from one lineage to another (trans-differentiation) or converted back to uncommitted multipotent cells (dedifferentiation) by transcription factors differentially-expressed in HSC and MPP, but not in mature fully-committed lineage cells. To identify transcription factors differentiallyexpressed in HSC and MPP, we sorted $\mathrm{Lin}^{-} \mathrm{CD} 48^{-} \mathrm{c}-\mathrm{kit}^{+} \mathrm{Sca}-1^{+} \mathrm{CD} 150^{+} \mathrm{HSC}, \mathrm{Lin}^{-} \mathrm{CD} 48^{-} \mathrm{c}-$ $\mathrm{kit}^{+} \mathrm{Sca}-1^{+} \mathrm{CD} 150^{-} \mathrm{MPP}, \mathrm{Ter} 119^{-} \mathrm{Gr}^{-} \mathrm{Mac1}^{+}$myeloid cells, Ter119- $\mathrm{CD} 19^{-} \mathrm{Mac1}^{-} \mathrm{CD}{ }^{+} \mathrm{T}$ 
lymphoid cells and Ter119- $\mathrm{Mac}^{-} \mathrm{CD}^{-} \mathrm{CD} 19^{+} \mathrm{B}$ lymphoid cells from bone marrow nucleated cells of eight-week-old female C57BL/6 mice and conducted gene expression analysis by RNA-Seq. Genes were designated as differentially-expressed in HSC and MPP if they demonstrated $>2$ fold higher relative expression in HSC and MPP than in lineagecommitted cells $(\mathrm{P}<0.05)$. The genes that met these criteria were screened for a match in the transcription factor database (http://genome.gsc.riken.jp/TFdb/tf_list.html), and this screen identified 15 candidate transcription factors expressed in HSC and MPP but not lineage-committed cells (Fig. 1a).

Each of these 15 transcription factors was cloned into a retroviral expression cassette, and a retroviral mixture containing clones for all 15 transcription factors (15-TF) was transduced into sorted Ter $119^{-} \mathrm{Mac}^{-} \mathrm{CD}^{-} \mathrm{CD} 4^{-} \mathrm{CD} 8^{-} \mathrm{B} 220^{+} \mathrm{CD} 19^{+} \mathrm{CD}^{-} 3^{+} \mathrm{IgM}^{-}$pro-pre-B progenitors (Supplementary Fig. 1a). We used pro-pre-B cells as targets for reprogramming because they carry genomic immunoglobulin $(\mathrm{Ig})$ heavy chain $\mathrm{V}(\mathrm{D}) \mathrm{J}$ rearrangements that serve as natural genetic barcodes, and they have weak epigenetic barriers for reprogramming ${ }^{16}$. Virus titres in the mixture were adjusted to homogenous $\mathrm{MOI}(\mathrm{MOI}=0.69)$ to yield a transduction rate for each clone of $\sim 50 \%$ (Fig. 1b). Five million $\mathrm{GFP}^{+}$pro-pre-B cells (C57BL/6 background, four-week-old female mice) transduced with the 15-TF retrovirus mixture were transplanted into sublethally-irradiated (6.5 Gy) CD45.2 C57BL/6 mice (hereafter 15-TF mice) and the percentages of $\mathrm{GFP}^{+}$cells in thymus and bone marrow (BM) of recipients were analysed by flow cytometry four weeks post-transplantation (Supplementary Fig. 1b). A significant proportion of $\mathrm{GFP}^{+}$thymocytes were observed in $50 \%$ of the $15-\mathrm{TF}$ mice, but no $\mathrm{GFP}^{+}$ thymocytes were observed in recipient mice transplanted with control-virus-transduced propre-B cells (Fig. 1c). Flow cytometry analysis of $\mathrm{GFP}^{+}$thymocytes revealed the presence of $\mathrm{CD} 44^{+} \mathrm{CD} 25^{-} \mathrm{CD}^{-}{ }^{-} \mathrm{CD} 8^{-}$(double negative) DN1 thymocytes, $\mathrm{CD} 44^{+} \mathrm{CD} 25^{+} \mathrm{DN} 2$, CD $44^{-} \mathrm{CD} 25_{+}$DN3 and CD44_CD25_ DN4, CD4 ${ }^{+} \mathrm{CD} 8^{+}$(double-positive) DP, CD4 ${ }^{+} \mathrm{CD} 8^{-}$ SP T lymphocytes and $\mathrm{CD}^{+} \mathrm{CD}^{-} \mathrm{SP} T$ lymphocytes (Fig. 1d).

Flow cytometry analysis on the $\mathrm{B}$ cell compartment of $15-\mathrm{TF}$ pro-pre-B cell recipient mice $\left(\mathrm{CD} 45.1^{+} \mathrm{C} 57 \mathrm{BL} / 6\right)$ showed that the percentages of $\mathrm{GFP}^{+} \mathrm{B} 220^{+} \mathrm{CD} 19^{+} \mathrm{IgD}^{\mathrm{lo}} \mathrm{IgM}^{\mathrm{hi}}$ immature B cells in spleen and peripheral blood (Supplementary Fig. 1c, d), and GFP ${ }^{+} \mathrm{B} 220^{+} \mathrm{CD} 19^{+} \mathrm{CD} 3^{+} \mathrm{IgM}^{-}$pro-pre-B cells in $\mathrm{BM}$ (Supplementary Fig. 1e, f) were markedly lower, while the percentages of $\mathrm{GFP}^{+} \mathrm{CD} 45.2^{+} \mathrm{B} 220^{+} \mathrm{CD} 19^{+} \mathrm{IgD}^{\mathrm{hi}} \mathrm{IgM}^{\mathrm{lo}}$ mature $\mathrm{B}$ cells in BM, spleen and peripheral blood (Supplementary Fig. 1c,d) were markedly higher compared to $\mathrm{GFP}^{-} \mathrm{CD} 45.2^{+}$counterparts, suggesting that the expression of the $15-\mathrm{TF}$ viral mix altered $\mathrm{B}$ cell development. These results indicate that forced expression of transcription factors can reprogram committed B cell progenitors into distinct subsets of $\mathrm{T}$ lymphocytes.

\section{Retro-expression of Hoxb5 reprograms B cells into T cells}

To identify which of the 15 candidate transcription factor(s) induces the $\mathrm{B}$ to $\mathrm{T}$ cell conversion, we sorted $\mathrm{GFP}^{+}$thymic thymocytes from 15-TF mice and used in single-cell PCR to identify retroviral sequences integrated in the genomic DNA of these cells. To avoid amplifying the endogenous genes, the PCR primer pairs flanked the introns of the 15-TF candidate genes (Supplementary Table. 1). All $70 \mathrm{GFP}^{+}$thymocytes analysed contained retrovirus-derived ectopic copies of Hoxb5 (Supplementary Table. 2). Further, Hoxb5- 
expressing retrovirus (retro-Hoxb5), a mixture of retroviral clones expressing the 14 other TFs, but lacking Hoxb5 (14-TF) or GFP-control virus were transduced into pro-pre-B cells that were retro-orbitally transferred into sublethally irradiated congenic mice (CD45.2 strain, C57BL/6 background). In all retro-Hoxb5-pro-pre-B cell recipient mice (retro-Hoxb5 mice), over $65 \%$ of single thymic nucleated cells were Ter $119^{-} \mathrm{Mac}^{-}{ }^{-} \mathrm{CD} 19^{-} \mathrm{GFP}^{+}$, while Ter119- $\mathrm{Mac}^{-} \mathrm{CD}^{-} 9^{-} \mathrm{GFP}^{+}$single thymic nucleated cells were not detected in mice transferred with 14-TF or pro-pre-B cells (Fig. 2a). The proportion of GFP ${ }^{+}$Ter119- $\mathrm{Mac}^{-} \mathrm{CD} 19^{-}$cells increased from about $10 \%$ two weeks after transplantation to $>40 \%$ from 3-4 weeks post-transplantation, and then gradually decreased from 5 to 8 weeks post-transplantation, until they were no longer detected (Fig. $2 \mathrm{~b}, \mathrm{c}$ ). $\mathrm{GFP}^{+} \mathrm{CD}^{+}{ }^{+}$and $\mathrm{CD} 8^{+}$ mature $\mathrm{T}$ cells were detected in peripheral blood (PB), spleen and lymph nodes (LN) of retro-Haxb5 mice at 4 and 8 weeks post-transplantation, followed by a decrease and disappearance in PB 12 weeks post-transplantation (Fig. 2d). GFP ${ }^{+} \mathrm{T}$ cells included both TCR- $\beta^{+}$and TCR- $\gamma \delta^{+}$cells (Fig. $2 \mathrm{~d}$ ).

To address the impact of constitutive expression of $H o x b 5$ on T cell development, we crossed ROSA26-Hoxb5 transgenic mice $\left(H o x b 5^{\mathrm{LSL} /+}\right)$ with Vav-Cre mice to generate Hox $b 5^{\mathrm{LSL} /+}$ Vav-Cre mice (Supplementary Fig. 2a) that constitutively express Hoxb5 in all hematopoietic cells, including T lymphocytes. Hox $b 5^{\mathrm{LSL} /+}$ Vav-Cre mice had comparable ratios of ETP in $\mathrm{BM}$ and thymus, $\mathrm{DN}$ and $\mathrm{DP}$ cells in thymus, and $\mathrm{CD} 4^{+}$and $\mathrm{CD} 8^{+} \mathrm{T}$ lymphocytes in $\mathrm{PB}$, spleen, $\mathrm{LN}$ and BM in comparison with their counterparts in $H o x b 5^{\mathrm{LSL}+}$ control mice (Supplementary Fig. 2d-f).

To further examine the impact of Hoxb5 expression on hematopoiesis, we retro-orbitally injected half million total BM cells from either Hoxb $5^{\mathrm{LSL} /+}$ Vav-Cre or Hoxb5 ${ }^{-/-}$mice $\left(\mathrm{CD} 45.2^{+}\right)$with equal number wild-type total BM cell competitors $\left(\mathrm{CD} 45.1^{+}\right)$into lethally irradiated $(9.0 \mathrm{~Gy}) \mathrm{CD} 45.1^{+}$recipients. Hox $b 5^{\mathrm{LSL} /+}$ Vav-Cre (Supplementary Fig. 3a-f) and $\mathrm{Hox} 5^{-1-}$ (Supplementary Fig. 3g-l) HSC differentiated over similar time frames into multilineages with similar patterns compared to wild-type competitors. Thus, overexpression of Hoxb5 has minimal impact on hematopoiesis.

To confirm the B cell origin of the GFP ${ }^{+} \mathrm{T}$ cells, we examined the Ig heavy chain V(D)J rearrangements ${ }^{17,18}$ in $\mathrm{GFP}^{+}$splenic T cells in retro-Hoxb5 mice. Single-cell PCR analysis with rearranged Ig heavy chain V(D)J and TCR $\beta$ V(D)J amplifying primers (Supplementary Table 3) indicated that individual splenic $\mathrm{GFP}^{+} \mathrm{T}$ lymphocytes simultaneously contained BCR Ig heavy chain V(D)J and TCR $\beta$ V(D)J rearrangements (Supplementary Table 4, Supplementary Fig. 5a), indicating their B cell origin. In addition, the distinct TCR $\beta$ V(D)J sequences of individual $\mathrm{GFP}^{+} \mathrm{T}$ cells indicated they were polyclonal (Supplementary Table

4). These results indicate that the expression of Hoxb5 is sufficient to convert pro-pre-B cells to $\mathrm{T}$ lymphocytes (iT cells) in vivo.

\section{B cell-specific expression of Hoxb5 converts B to T cells}

In order to exclude the possibility that retroviral transduction and integration plays a role in the B to T cell conversion, we crossed ROSA26-Hoxb5 knock-in transgenic mice Hox $b 5^{\mathrm{LSL} /+}$ with CD19-Cre mice to generate Hoxb $5^{\mathrm{LSL} /+} \mathrm{CD} 19-\mathrm{Cre}$ mice (CD19-Hoxb5 mice hereafter) (Supplementary Fig. 2a), in which a GFP reporter in the Hoxb5 cassette can 
be used to track Hoxb5 expression. Approximately $90 \%$ of $\mathrm{CD} 19^{+} \mathrm{B}$ lymphocytes in the blood of CD19-Hoxb5 mice expressed GFP-Hoxb5, while CD3 ${ }^{+}$T lymphocytes or Mac1 ${ }^{+}$ myeloid cells in the PB were $\mathrm{GFP}^{-}$(Fig. 3a). However, $\mathrm{GFP}^{+} \mathrm{T}$ lymphocytes were detected in the thymus of 4, 8 and 12-week-old CD 19-Hoxb5 mice (Fig. 3b). Further, B cells overexpressing Hoxb5 showed a comparable developmental pattern as their counterparts from littermate control (Supplementary Fig. 2b, c), indicating that induction of Hoxb5 at the pro-pre-B cell stage had minimal impact on B cell development. In comparison with the retro-Hoxb5 mice, $\mathrm{GFP}^{+} \mathrm{T}$ lymphocytes were much less abundant in CD19-Hoxb5 mice (Fig. 3b), which could reflect the competition of endogenous $\mathrm{T}$ lymphocytes generated from HSC under homeostasis. To test this possibility, CD19-Hoxb5 $\mathrm{GFP}^{+}$pro-pre-B cells isolated from BM were transplanted into sublethally-irradiated wild-type congenic recipient mice. Four weeks after transplantation, $>30 \%$ of T lymphocytes in thymus were $\mathrm{GFP}^{+}$(Fig. 3c). In addition, $>10 \%$ of $\mathrm{T}$ lymphocytes in spleen (Fig. 3d), and $>7 \%$ in LN (Fig. 3e) were GFP ${ }^{+}$. Further, splenic $\mathrm{GFP}^{+} \mathrm{CD}^{+} \mathrm{T}$ cells possessed both Ig heavy chain $\mathrm{V}(\mathrm{D}) \mathrm{J}$ and $\mathrm{TCR} \beta \mathrm{V}(\mathrm{D}) \mathrm{J}$ rearrangements (Supplementary table 4, Supplementary Fig. 5b). Taken together, these results indicate that transgenic expression of Hoxb5 reprograms B into T cells in vivo.

\section{Hoxb5-induced $\mathrm{T}$ cells are functionally equivalent to wild-type $\mathrm{T}$ cells}

To evaluate the function of mature $\mathrm{GFP}^{+}$iT lymphocytes, we transplanted three million retro-Hoxb5 pro-pre-B cells into sublethally-irradiated (3.5 Gy) $\mathrm{Rag}^{-{ }^{--}}$recipient mice, which lack B and T cells. $\mathrm{GFP}^{+}$iT lymphocytes were detected in the PB of Rag1 $1^{-/-}$ recipient mice 3 weeks after transfer (Supplementary Fig.4b). Splenic GFP ${ }^{+} \mathrm{T}$ cells isolated from Rag $1^{-/-}$mice transplanted with retro-Hoxb5 pro-pre-B cells (Hoxb5-Rag ${ }^{-/-}$mice hereafter) 4 weeks post-transplantation and incubated in vitro with CD3 and CD28 antibodies for six days, produced interleukin 2 (IL-2), IL-10, interferon- $\gamma$ (IFN- $\gamma$ ) and tumor necrosis factor (TNF) in similar amounts as wild-type splenic T cells (Fig. 4a,b). Further, incubation of Hoxb5-Rag $1^{-/}$splenocytes with inactivated allogeneic splenocytes from $\mathrm{BALB} / \mathrm{c}$ mice induced rapid proliferation of $\mathrm{GFP}^{+} \mathrm{CD}^{+} \mathrm{T}$ cells from Hoxb5-Rag $1^{-/-}$ mice (Fig. 4c,d).

To examine the functions of Hoxb5-induced T cells in vivo, we grafted skin from BALB/c mice into C57BL/6 Hoxb5-Rag1 ${ }^{-/}$mice three weeks after the transfer of CD19-Hoxb5 propre-B cells. The allogeneic skin grafts were rapidly rejected in Hoxb5-Rag $1^{-1-}$ mice about 10 days after transplantation, as indicated by bulged, ulcerative and necrotic lesions formed at the graft site (Supplementary Fig. 4a). $\mathrm{GFP}^{+} \mathrm{CD}^{+}$iT lymphocytes infiltrated the dermis of the allogeneic skin (Fig. 5d). To evaluate the memory response of iT cells, we performed secondary allogeneic skin graft assay 8 weeks after the primary allogeneic skin transplantation. The median survival of the secondary allogeneic skin grafts $(7.5 \mathrm{~d})$ was significantly shorter than that of the primary allogeneic skin grafts (10.5 d) (Fig. 5a). Flow cytometry indicated the presence of $\mathrm{CD} 44^{\mathrm{hi}} \mathrm{CD} 69^{+} \mathrm{CD} 8^{+}$and $\mathrm{CD} 4^{+} \mathrm{T}$ lymphocytes (Fig. $5 \mathrm{~b}$ ), as well as IL- $17^{+}$and IFN- $\gamma^{+} \mathrm{CD} 4^{+} \mathrm{T}$ lymphocytes and IFN- $\gamma^{+} \mathrm{CD} 8^{+} \mathrm{T}$ lymphocytes in rejected primary and secondary grafts (Fig. 5c). These results indicate that Hoxb5induced $\mathrm{T}$ lymphocytes are activated in vitro and mediate allogeneic skin graft rejection and sustained immunological memory, suggesting a typical adaptive immune response. 


\section{Transient expression of Hoxb5 reprograms B into T lymphocytes}

Next, we constructed and characterized an inducible transgenic model (Tet-Hoxb5, CD45.2 strain, C57BL/6 background), in which ectopic Hoxb5 can be induced by doxycycline. The Hoxb5 expression cassette in the Tet-Hoxb5 mice includes a BFP reporter gene fused to the $\mathrm{C}$ terminus of $H o x b 5$ by a p2A splicing element (Supplementary Fig. 6a), which allows tracking of cells that conditionally express Hoxb5. In Tet-Hoxb5 mice maintained on 1 $\mathrm{mg} / \mathrm{mL}$ doxycycline in the drinking water for one week, $\mathrm{BFP}^{+}$pro-pre-B cells were detected in the BM of eight-week-old Tet-Hoxb5 mice (Fig. 6a). Next, BM BFP ${ }^{+}$pro-pre-B cells sorted from Tet-Hoxb5 mice (CD45.2) were transferred into NOD-SCID (CD45.1) recipient mice (Tet-Hoxb5-NOD-SCID mice hereafter) on $1 \mathrm{mg} / \mathrm{mL}$ doxycycline in the drinking water starting one day prior to and up to 4 weeks post-transplantation. Four weeks posttransfer, $\mathrm{BFP}^{+} \mathrm{CD} 45.2^{+} \mathrm{Ter} 119^{-} \mathrm{CD} 19^{-} \mathrm{Mac}^{-} \mathrm{CD}^{+} \mathrm{T}$ lymphocytes were detected in $\mathrm{PB}$ and spleen of recipient mice, though over $95 \%$ of donor-derived CD45.2 $2^{+} \mathrm{T}$ cells were $\mathrm{BFP}^{-}$ (Supplementary Fig. 6c). When Tet-Hoxb5-NOD-SCID mice were maintained on doxycycline for two weeks post-transplantation and then switched to water without doxycycline (Supplementary Fig. 6b), all T lymphocytes in $\mathrm{PB}$ and spleen were $\mathrm{BFP}^{-}$four weeks post-transplantation (Supplementary Fig. 6d). Further, all $\mathrm{BFP}^{+} \mathrm{T}$ cells, but not $\mathrm{BFP}^{-}$ T cells, expressed Hoxb5 in Tet-Hoxb5-NOD-SCID mice (Supplementary Fig. 6e). Donorderived CD45.2 $2^{+} \mathrm{T}$ lymphocytes were still detected in the PB, LN, spleen, and thymus of Tet-Hoxb5-NOD-SCID mice 4, 8 and 12 weeks post-transplantation, then sharply decreased in PB (Fig. 6b, 6c).

We next investigated whether Hoxb5 induced the de-differentiation of pro-pre-B cells into multipotent progenitors. We analysed the upstream multi-lineage progenitor cells, including $\mathrm{CD} 2^{-} \mathrm{CD} 3^{-}{ }^{-}{ }^{-} 4^{-} \mathrm{CD} 8^{-} \mathrm{B} 220^{-} \mathrm{Mac}^{-}{ }^{-} \mathrm{Gr} 1^{-} \mathrm{Ter} 119^{-}\left(\mathrm{Lin}^{-}\right) \mathrm{c}^{-\mathrm{kit}}{ }^{+} \mathrm{Sca}-1^{+} \mathrm{LSK}$ cells, which include HSC, MPP and LMPP subsets and $\mathrm{Lin}^{-} \mathrm{CD} 127^{+} \mathrm{c}-\mathrm{kit}^{\mathrm{int}} \mathrm{Sca}-1^{\text {int }}$ common lymphoid progenitor cells (CLP) in the BM of 15-TF mice, retro-Hoxb5 mice, CD19-Hoxb5 pro-pre-B cell recipient mice, and Tet-Hoxb5 pro-pre-B cell recipient mice. We did not detect donorderived LSK and CLP in the BM of these mice (Supplementary Fig. 7a-d). In addition, the BFP $^{-}$T cells in Tet-Hoxb5-NOD-SCID mice simultaneously carried BCR Ig H V(D)J and TCR $\beta$ V(D)J rearrangements (Supplementary table 4, Supplementary Fig. 5c), indicating their B cell origin. As such, transient expression of $H o x b 5$ in pro-pre-B cells is sufficient for stable conversion of B to T cells in vivo, and sustained expression of Hoxb5 is dispensable after the target cells commit to lineage conversion.

\section{Hoxb5 converts B cells into ETPs}

To investigate whether Hoxb5 directly reprograms pro-pre-B cells into ETPs (iETPs), we examined the dynamics of $\mathrm{Lin}^{-} \mathrm{CD} 44^{+} \mathrm{c}-\mathrm{kit}^{\mathrm{hi}} \mathrm{CD} 25^{-}$iETPs (Fig. 7a) in the BM and thymus of retro-Hox 55 mice. GFP ${ }^{+}$iETPs were detected in the BM of retro-Hox $b 5$ mice from week 2 to 6 post-transplantation (Fig. 7b), with maximum abundance approximately 3 weeks posttransplantation, and in thymus from week 2 to 6 post-transplantation (Fig. 7b), with maximum abundance at approximately 4 weeks post-transplantation. RNA-Seq analysis was performed on $\mathrm{GFP}^{+} \mathrm{Lin}^{-} \mathrm{CD} 44^{+} \mathrm{c}-\mathrm{kit}^{\mathrm{hi}} \mathrm{CD} 25^{-}$iETPs sorted from BM and thymus of retroHoxb5 mice or $\mathrm{GFP}^{-} \mathrm{Lin}^{-} \mathrm{CD} 44^{+} \mathrm{c}-\mathrm{kit}^{\mathrm{hi}} \mathrm{CD} 25^{-}$ETPs from the thymus of eight-week-old wild-type mice (CD45.2 strain, C57BL/6 background). Unsupervised hierarchical clustering 
analysis revealed that $\mathrm{GFP}^{+}$iETPs in the thymus mapped closely to wild-type thymic ETPs (Fig. 7c). In contrast, $\mathrm{GFP}^{+}$iETPs in the BM clustered closely to wild-type pro-pre-B cells (Fig. 7c). Of note, some B cell-related genes were still expressed in $\mathrm{BM} \mathrm{GFP}^{+} \mathrm{iETPs}$, including Cd19, Cd79a, Ebf1 and Pax5 (Fig. 7d), suggesting that BM GFP ${ }^{+}$iETPs were in the process of lineage conversion. GFP ${ }^{+}$iETPs in the thymus had entirely repressed the expression of B lineage regulator Pax 5 and Ebfl (Fig. 7d), consistent with previous reports that repression of Pax 5 and $E b f 1$ is crucial for B to T cell conversion ${ }^{19,20}$. These data support the hypothesis that Hoxb5-induced conversion of B cells to ETPs begins in BM, while reprogramming is completed in thymus.

We further examined the gene expression pattern of thymic GFP ${ }^{+}$iETP progeny in retroHoxb5 mice at DN1, DN2, DN3, DN4, DP, CD4 SP and CD8 SP stages of development as defined above. Unsupervised hierarchical clustering analysis revealed that $\mathrm{GFP}^{+} \mathrm{iDN} 1$, iDN2 and iDN3 cells mapped closely to wild-type DN1, DN2, and DN3 thymocytes, respectively, and $\mathrm{GFP}^{+}$iDN4 cells mapped closely to iCD8 SP and wild-type DN4 cells (Fig. 7e). GFP iDPs mapped closely to wild-type DP cells, whereas GFP ${ }^{+}$iCD8 SP and iCD4 SP cells were close to wild-type CD8 SP and CD4 SP, respectively (Fig. 7e). Therefore, the Hoxb5-induced thymocyte subsets resemble their wild-type counterparts at the transcriptome level.

To confirm the functionality of the iETPs in the thymus, we performed a secondary transplantation assay in which total thymocytes from the retro-Hoxb5 mice were transferred into the thymus of sublethally irradiated CD45.2 congenic mice (C57BL/6 background). Three weeks after secondary transplantation, $\mathrm{GFP}^{+} \mathrm{CD}^{-} \mathrm{CD}^{-} \mathrm{CD}^{-} 4^{-} \mathrm{CD} 25^{+}$iDN3 and $\mathrm{GFP}^{+} \mathrm{CD} 4^{-} \mathrm{CD}^{-} \mathrm{CD}^{-} 4^{-} \mathrm{CD} 25^{-}$iDN4, but not $\mathrm{GFP}^{+} \mathrm{CD} 4^{-} \mathrm{CD} 8^{-} \mathrm{CD} 44^{+} \mathrm{CD} 25^{-} \mathrm{DN} 1$ or CD4- $4^{-} D 8^{-} \mathrm{CD}_{4} 4^{+} \mathrm{CD} 25^{+}$iDN2 cells were detected in the thymus of secondary recipients. We also detected mature $\mathrm{GFP}^{+} \mathrm{CD}^{+}$iT cells in the spleen (1.9\%), $\mathrm{LN}(3.7 \%)$ and $\mathrm{BM}$ $(2.8 \%)$ of the secondary recipients (Fig. $7 \mathrm{f}$ ), with phenotypes and tissue distribution patterns similar to wild-type mice (Supplementary Fig. 7e-g), suggesting that iETPs normally differentiated into $\mathrm{T}$ cells in secondary recipient mice. Thus, Hox 55 converts B cells into functional ETPs.

\section{Hoxb5 targets B, T cell-regulators and chromatin modifiers}

To investigate the target genes of Hoxb5 in pro-pre-B cells, we performed RNA-Seq analysis of retro-Hoxb5 or empty-vector transduced pro-pre-B cells after 3-day culture with IL-7, Flt31, and SCF. Differential expression gene (DEG) analysis using the DESeq2 R package indicated 358 genes significantly up-regulated and 422 genes significantly down-regulated in retro-Hoxb5 relative to empty vector pro-pre-B cells (> 2 fold, $\mathrm{p}$ adj < 0.05) (Fig. 8a, Supplementary Table. 5). Using a lower threshold for DEG analysis (> 1.2 fold, $\mathrm{p}$ adj < 0.01), we found 3700 DEGs (Supplementary Table. 6) as potential Hoxb5 targets, of which 232 (Supplementary Table. 7) were identified previously as transcription factors (http:// genome.gsc.riken.jp/TFdb/tf_list.html).

Gene ontology analysis of these 232 transcription factors indicated enrichment of genes involved in chromatin modification and remodeling, myeloid and lymphoid cell differentiation and $\mathrm{T}$ cell differentiation in retro-Hoxb5 pro-pre-B cells compared to empty 
vector (Fig. 8b). Expression of Hoxb5 in pro-pre-B cells altered the expression of many genes related to chromatin modification, including the epigenetic modifier Hdac9, Ezh1, $L d b 1, C b x 8$ and $A s x 11$ (Fig. 8c), consistent with the alteration in the gene expression pattern during $\mathrm{B}$ to $\mathrm{T}$ cell fate reprogramming. Importantly, transcription factors essential for early B cell development, such as Ebf1, Bcl11a, Foxp1 and Foxo1 ${ }^{21-24}$ were repressed by Hoxb5 (Fig. 8d), while transcription factors critical for T cell development or function, such as Nfatc1, Tcf12, Lmo2, and Prdm1 ${ }^{25-28}$ were activated by Hoxb5 in pro-pre-B cells (Fig. 8d). In addition, the B cell-specific marker $C d 19$ was reduced, whereas the $\mathrm{T}$ cell markers $I 117 \mathrm{ra}$ and II2ra were increased by Hoxb5 expression in pro-pre-B cells (Supplementary Table 6). GSEA analysis indicated that target genes for Ikzf1 (Ikaros) and Pax5, which are essential for B cell development ${ }^{29-31}$, were significantly repressed in retro- $H o x b 5$ pro-pre-B cells compared to empty vector (Fig. 8e,f). Furthermore, target genes of the epigenetic modifier $K m t 2 a(M I I)$, a histone methyltransferase important for HSC self-renewal ${ }^{32,33}$, were significantly repressed in retro-Hoxb5 pro-pre-B cells compared to empty vector (Fig. 8g). These results indicate that $H o x b 5$ expression in pro-pre-B cells represses the expression of $\mathrm{B}$ cell lineage-specific transcription factors and signature genes, enhances the expression of transcription factors and genes relevant to T cell development, and alters the expression of genes related to chromatin and epigenetic modifications.

To identify the direct targets of Hoxb5 in pro-pre-B cells, we performed CHIP-Seq analysis in retro-Hoxb5 pro-pre-B cells. Due to the lack of CHIP-Seq grade anti-Hoxb5 antibodies, pro-pre-B cells from Rosa26 $6^{\mathrm{Bir} / \mathrm{BirA}}$ transgenic mice (C57BL/6), which constitutively express BirA enzyme that can biotinylate bio-tagged protein, were transduced with a Hoxb5biotin acceptor sequence fusion protein (Bio-Hoxb5) (Supplementary Fig. 8a), resulting in the biotinylation of Bio-Hoxb5 protein (Supplementary Fig. 8b). Of note, expression of BioHox $b 5$ protein did not alter the ability of Hoxb5 to reprogram B into T lymphocytes in vivo (Supplementary Fig. 8c,d). B220 ${ }^{+}$B cells isolated from the BM of six-week-old Rosa26 $6^{\text {BirA/BirA }}$ mice were transduced with GFP-Bio-Hoxb5-retrovirus, cultured for 3 days in vitro, and the viable $\mathrm{GFP}^{+}$Bio-Hoxb5 pro-pre-B cells were sorted for streptavidinmediated ChIP-seq of Hoxb5. 163 peak-correlated TFs were identified in Bio-Hoxb5 propre-B cells (Supplementary Table. 8), which were overlapped with the DEGs from the transcriptome analysis (Supplementary Table. 6). Hoxb5 directly targeted the transcription factors Ebf1, Pax5, Bcl11a, Foxp1 and Foxo1 (Fig. 8h) ${ }^{21-24,34}$, which are essential for B cell development, and the transcription factor Lmo2, Nfatc1, Tcf12, Prdm1 and Runx2 (Fig. 8h, Supplementary Fig. 8e, and Supplementary Table. 8), which play important roles in regulating $\mathrm{T}$ cell functions $25-28,35$. In addition, Hoxb5 directly targeted the chromatin modifier Kmt2a (MII), Hdac9, Ldb1 and Smarca5 (Fig. 8h, Supplementary Fig. 8e) ${ }^{36-39}$. As such, Hoxb5 directly targets B cell master regulators, T cell regulators and crucial chromatin modifiers in pro-pre-B cells, ultimately driving B to $\mathrm{T}$ cell fate conversion.

\section{DISCUSSION}

Here we show that forced expression of Hoxb5 in pro-pre-B cells was sufficient to convert murine B cells into T cells in vivo. After transplantation into sublethally-irradiated recipient mice, Hoxb5-expressing pro-pre-B cells gave rise to ETPs in BM, which subsequently matured into fully functional polyclonal $\mathrm{T}$ lymphocytes in the thymus of recipient mice. This 
cell fate conversion was the consequence of Hoxb5-mediated repression of B cell master regulators, activation of $\mathrm{T}$ cell regulators and regulation of chromatin and epigenetic modifiers.

Under homeostasis, the efficiency of Hoxb5-induced B to T cell conversion was very low. HSC-derived natural T lymphopoiesis could outperform Hoxb5-induced T lymphopoiesis. However, in the adoptive transfer experiments with irradiated mice, the irradiation at least partially eradicated HSC and progenitors and transiently impaired endogenous T lymphopoiesis, allowing for the pro-pre-B-derived iT lymphopoiesis to occur in the BM microenvironments. Hoxb5-mediated B to T cell conversion started in the BM and completed in the thymus. Detection of reprogrammed intermediates in the BM indicates that the BM niche plays an essential role and is required during $\mathrm{B}$ to $\mathrm{T}$ cell reprogramming in recipient mice. The complete silencing of B cell master regulator Ebf1 and Pax 5 was only observed in thymic iETPs, suggesting that the thymic niche also plays a crucial role in B to $\mathrm{T}$ cell reprogramming. Moreover, the thymic stromal niches guarantee the essential processes of negative and positive selections of the iT lymphopoiesis ${ }^{40}$, and thus reduce the risk of generating autoreactive iETP derivatives.

In the $\mathrm{B}$ to $\mathrm{T}$ cell converting system induced by retro-Hox 65 expression, it can be estimated that one million retrovirally-transduced Hoxb5-expressing pro-pre-B cells gave rise to tenthousands of ETPs in vivo, suggesting that reprogramming efficiency by Hoxb5 may have been as low as $1 \%$. Thus, a minority of pro-pre-B cells overexpressing Hoxb5 was reprogrammed into iETP cells, but the majority of them could differentiate normally into B cells. The transcription factor Cebpa can reprogram B into macrophages at an efficiency of $100 \%{ }^{4}$, which could indicate that co-factor(s) that enhances the efficiency of B to T cell reprogramming by Hoxb5 may exist. In addition, deletion of endogenous Hoxb5 did not affect hematopoiesis, including B and T lymphopoiesis. Consistently, mice lacking the entire Hoxb gene clusters have normal hematopoiesis ${ }^{15}$. One possible explanation for the dispensability of Hoxb5 during normal lymphopoiesis is the existence of potential compensatory gene(s). Identification of the potential co-factor(s) or compensatory gene(s) of Hoxb5 might improve our understanding of Hoxb5-induced B to T cell conversion.

It should be emphasized that forced expression of Hoxb5 in pro-pre-B cells, using the procedure described here, yields fully functional T lymphocytes in vivo, whose transcriptomes, hierarchical differentiation, tissue distribution and immune functions closely resemble those of endogenous murine $\mathrm{T}$ cells. Thus, the approach has advantages over alternative approaches previously explored, including direct manipulation of expression of Pax 5 and $E b f 1^{10,11}$ This could reflect the fact that endogenous Hoxb5 is expressed in HSC and MPP ${ }^{14}$ but is not expressed in committed B or T cells. Thus, this research could lead to new insights for understanding immune system lineage conversion.

\section{METHODS}

Mice.

C57BL/6 (CD45.1), and CD19-Cre (C57BL/6, CD45.2) mice were purchased from the Jackson Laboratory. C57BL/6 (CD45.2), NOD-SCID and BALB/c mice were purchased 
from Bejing Vital River Laboratory Animal Technology Ltd. Rag1 ${ }^{-/-}$mice (C57BL/6) were gifted from Dr. Zhihua Liu from Institute of Biophysics (CAS, China) and from Model Animal Research Center of Nanjing University. Hox $b 5^{\mathrm{LSL} /+}$ and Tet-Hoxb5 mice were generated by targeting a mouse ES line (C57BL/6 line, Beijing Biocytogen Co., Ltd.) through homologous recombination at the ROSA26 locus. Hox $b 5^{\mathrm{LSL} /+}$ mice were subsequently bred with CD19-Cre or Vav-cre mice to generate $H o x b 55^{\mathrm{LSL} /+} \mathrm{CD} 19$-cre and $H o x b 5^{\mathrm{LSL} /+}$ Vav-cre mice. Hox $b 5^{-1-}$ mice were generated by direct targeting the zygotes of C57BL/6 (CD45.2) mice via TALEN to delete 37-bp from Exon 1 of Hoxb5 on Chr11. Mice were housed in the SPF grade animal facility of the Guangzhou Institution of Biomedicine and Health, Chinese Academy of Science (GIBH, CAS, China). All animal experiments were approved by the Institutional Animal Care and Use Committee of Guangzhou Institutes of Biomedicine and Health (IACUC-GIBH).

\section{Recombinant vectors, virus packaging, and transduction of pro-pre-B cells.}

The cDNA of each factor was inserted into pMYs-IRES-EGFP to generate a recombinant vector (RTV-021, Cell Biolabs, INC). Each recombinant vector or empty vector control was transduced (Calcium Phosphate Transfection method) into Plat-E cells (containing retrovirus packaging elements) to produce high-titer, replication-incompetent viruses. The titer of each virus was adjusted to $0.69 \mathrm{MOI}$ using a NIH/3T3 cell line as described ${ }^{41}$.

Subsequently, the transduction efficiency of each virus can reach $\sim 50 \%$ in pro-pre-B cells in our experimental setting.

Four to six-week-old donors (C57BL/6, CD45.2) were sacrificed and BM cells were collected. After lysis of red blood cells, the nucleated cells were blocked by Fc blocker, incubated with biotin-conjugated anti-B220 antibody, and enriched by Anti-Biotin MicroBeads by AutoMACS Pro (Miltenyi Biotec). pro-pre-B cells (Ter119- $\mathrm{Mac}^{-} \mathrm{CD}^{-} \mathrm{CD}^{-} \mathrm{CD} 8^{-} \mathrm{B} 220^{+} \mathrm{CD} 19^{+} \mathrm{CD} 93^{+} \mathrm{IgM}^{-}$) were sorted from the enriched $\mathrm{B} 220^{+}$cells by Moflo Astrios (Beckman Coulter) and then cultured in the medium (15\%FBS, $100 \mathrm{mM}$ GlutaMAX, 10-4 M $\beta$-ME, $10 \mathrm{ng} / \mathrm{ml} \mathrm{mSCF}, 10 \mathrm{ng} / \mathrm{ml}$ Flt3L, $10 \mathrm{ng} / \mathrm{ml}$ IL7) for 12-16 hours prior to virus transduction. Pro-pre-B cells ( 1 million/ml) were transduced with the mixed viruses having adjusted titers by two-round spin-infection ( $800 \mathrm{~g}$, $90 \mathrm{~min}, 35^{\circ} \mathrm{C}$ ).

\section{Transplantation.}

For pro-pre-B cell transplantation, one to 5 million sorted pro-pre-B cells were injected into the retro-orbital veins of sublethally (C57BL/6 recipients, 6.5 Gy; NOD-SCID recipients, 2.25 Gy; Rag $1^{-/-}$recipients, 3.5 Gy. RS2000, Rad Source Inc) irradiated recipients. Mice were fed with trimethoprim-sulfamethoxazole-treated water for two weeks to prevent infection. For competitive bone marrow transplantation assay, half million total bone marrow cells from either Hoxb5 $5^{\mathrm{LSL} /+}$ Vav-Cre or $\mathrm{Hoxb5}^{-1-}$ mice (CD45.2 ${ }^{+}$) with equivalent number of competitor cells $\left(\mathrm{CD} 45.1^{+}\right)$were retro-orbitally transplanted into lethally irradiated $(9.0$ Gy) individual CD45.1 $1^{+}$recipients. For intra-thymus transplantation, the surgery procedure was performed as described ${ }^{42}$. The amount of thymocytes equivalent to a quarter of a donor thymus were injected into sublethally irradiated congenic mice (CD45.2 $2^{+}$. 


\section{Flow cytometry analysis.}

Antibodies against CD2 (RM2-5), CD3 (145-2C11), CD4 (RM4-5), CD8a (53-6.7), Gr1 (RB6-8C5), Mac1 (M1/70), Ter119 (TER-119), IgM (II/41), Thy1.2 (53-2.1), B220 (6B2), c-kit (2B8), Sca-1 (E13-161.7), Fc $\gamma$ RII/III (2.4G2), CD25 (PC61), CD93 (PB.493), IgD (1.19), CD28 (37.51), Foxp3 (Fjk-16s), TCR $\beta$ (H57-597), TCR $\gamma \delta$ (GL3), CD21/35 (7G6), CD23 (B3B4), CD5 (53-7.3), CD69-PE (H1.2F3), IFN $\gamma$-APC (XMG1.2), IL-17-Percpcy5.5 (TC11-18H10.1), CD44 (IM7), CD127 (SB/199), Ly6D (49-H4) CD45.2 (104) CD45.1(A20) were from eBioscience or biolegend. DAPI, 7-AAD, and PI were used to stain dead cells. Flow cytometry was performed on LSR Fortessa (BD Biosciences) and data were processed by FlowJo software (Tree Star).

\section{Ig heavy chain $V(D) J$ rearrangements analysis.}

Semi-nested PCR for detecting Ig heavy chain V(D)J rearrangement was performed as described ${ }^{18}$. For single cell analysis, individual cells were sorted into PBS, lysed and subsequently amplified by Discover-sc single cell kit according to manufacturer's protocols (N601-02, Vazyme Biotech Co., Ltd). Amplification processes were carried out in two rounds: I, containing following 5'primers (VHJ558, 5'-ARGCCTGGGRCTTCAGTGAAG-3'; VHQ52, 5'-GCGAAGCTTCTCACAGAGCCTGTCCATCAC-3'.) and the JH4E primer (5'AGGCTCTGAGATCCCTAGACAG-3'), 200 ng DNA template, and with a $60{ }^{\circ} \mathrm{C}$ annealing/30s extension, and 20 cycle program; II, $0.25 \mu \mathrm{l}$ first round product was diluted and used as template. Then 35 cycles of amplification were performed with either primer pairs VHJ558 and the nested JH4A (5'-GGGTCTAGACTCTCAGCCGGCTCCCTCAGGG-3'), or primer pairs VHJ558 and the nested JH4A as described ${ }^{18}$.

\section{TCR beta chain V(D)J rearrangement analysis.}

Individual iT lymphocytes were sorted into PBS using AriaII sorter (BD Biosciences). The single cell genome was amplified by Discover-sc single cell kit according to manufacturer's protocols (N601-02, Vazyme Biotech Co., Ltd). PCR for detecting TCR $\beta$ V(D)J rearrangements was performed as described ${ }^{10,43}$ using the following primers: $V \beta 2$ (upstream): GGGTCACTGATACGGAGCTG, V $\beta 4$ (upstream): GGACAATCAGACTGCCTCAAGT, V $\beta 5.1$ (upstream): GTCCAACAGTTTGATGACTATCAC, V $\beta 8$ (upstream): GATGACATCATCAGGTTTTGTC, and jß2 (downstream): TGAGAGCTGTCTCCTACTATCGATT. After 35 cycles of amplification $\left(15 \mathrm{sec}\right.$ at $95^{\circ} \mathrm{C}, 20 \mathrm{sec}$ at $60^{\circ} \mathrm{C}, 1 \mathrm{~min}$ at $\left.72{ }^{\circ} \mathrm{C}\right), \mathrm{PCR}$ products were purified and cloned into pMD18-T vector (6011, TaKaRa). To confirm the identities of the PCR products, three clones of each recombinant were sequenced. The specific V(D)J rearrangements were further aligned by Igblast tool (https:// www.ncbi.nlm.nih.gov/igblast) ${ }^{44}$.

\section{RNA-Seq and data analysis.}

The cDNA of sorted 1000-cell aliquots were generated and amplified as described previously ${ }^{45}$. The qualities of the amplified cDNA were examined by qPCR analysis of housekeeping genes (B2m, Actb, Gapdh, and Ecf1al). Samples that passed quality control were used for sequencing library preparation by illumina Nextera XT DNA Sample Preparation Kit (FC-131-1096). All libraries were sequenced by illumina sequencers 
NextSeq 500 (illumina). The fastq files of sequencing raw data of the total 92 RNA-Seq samples were generated using illumina bcl2fastq software and were uploaded to Gene Expression Omnibus public database (GSE105057). Raw reads alignment and differential gene expression analysis were performed by Bowite2, DEseq2, Tophat2 and Cufflinks2.2.1 as reported ${ }^{46,47}$ The processed data were uploaded to the server of Gene Expression Omnibus (GSE105057). Unsupervised clustering analysis was performed using factextra (https://cran.r-project.org/web/packages/factoextra/index.html). Heatmaps were plotted using gplots (heatmap.2). Gene set enrichment analysis (GSEA) and gene ontology (GO) enrichment analysis (clusterProfiler R package) were performed as described ${ }^{48,49}$. The gene sets for GSEA analysis were from literature as follows: Ikzf1 (Ikaros) activated targets in pro-B ${ }^{29}$, Pax 5 activated targets in pro-B ${ }^{30,31}$ and $\mathrm{Kmt} 2 \mathrm{a}$ (MII) activated targets in CD $48^{-}$LSK $^{33}$.

\section{iT lymphocyte stimulation assay in vitro.}

The iT lymphocyte stimulation assay was performed according to the protocol described ${ }^{50}$. Briefly, the anti-CD3 antibodies (2C11) at a concentration of $50 \mu \mathrm{g} \mathrm{ml}^{-1}$ were coated to 96well plates $\left(100 \mu \mathrm{l} \mathrm{well}{ }^{-}\right)$overnight at $4{ }^{\circ} \mathrm{C}$, followed by two washes with PBS. Sorted splenic iT lymphocytes $\left(\mathrm{GFP}^{+} \mathrm{CD}^{+}\right)$were suspended at $10^{6}$ cells $/ \mathrm{ml}$ in RPMI 1640 medium (containing 10\% FBS and $100 \mathrm{mM}$ glutamine), and then seeded into the coated 96-well plates $\left(2 \times 10^{5}\right.$ cells/well). The RPMI 1640 culture medium containing $5 \mu \mathrm{g} / \mathrm{ml}$ of the antiCD28 antibody was used for cell culture. The cells were incubated for 6 days, and the supernatants were collected and analysed for IL2 (BMS601), IL10 (BMS614/2). IFN- $\gamma$ (BMS606) and TNF-a (BMS607/3) by ELISA according to manufacturer's guide (eBioscience).

\section{One way mixed lymphocyte reaction assay.}

Briefly, individual wells containing $5 \times 10^{5}$ iT responders (C57BL/6 background) from the allogeneic skin-rejected iT-Rag $1^{-/-}$mice and $5 \times 10^{5}$ irradiated (30 Gy) stimulators (BABL/c background) were incubated in 1640 medium containing 10\% FBS and $5 \mu \mathrm{M}$ cell proliferation dye Fluor670 (ebioscience, $65-0840$ ) at $37^{\circ} \mathrm{C}$ in $5 \% \mathrm{CO} 2$. The same responders incubated with medium without stimulators were used as a control. After three to four days, the cells were analysed by Flow cytometry.

\section{Allogeneic skin graft and immunofluorescence staining.}

The individual $\mathrm{Rag} 1^{-/}$mice were transplanted with 3 million pro-pre-B cells from Hox $b 5^{\mathrm{LSL} /+} \mathrm{CD} 19-\mathrm{Cre}$ or Hox $5^{\mathrm{LSL} /+}$ control mice. After three weeks, the allogeneic skin (BALB/c background) was transplanted using a procedure as described ${ }^{51}$. Grafts were considered rejection if there was a loss of distinct border, visible signs of ulceration and necrosis to $80 \%$ of the graft area. After six to ten days, the rejected skin tissues were removed for analysis. For activated $\mathrm{T}$ cells analysis, the cell suspensions were isolated as described ${ }^{52}$. The activated alloreactive iT lymphocytes were analysed by LSR Fortessa (BD Biosciences) by gating on $\mathrm{CD} 45.2^{+} \mathrm{Mac}^{-} \mathrm{CD} 19^{-} \mathrm{CD} 69^{+} \mathrm{CD} 44^{+} \mathrm{CD} 4^{+} / \mathrm{CD}^{+}$. Data were further processed by FlowJo software (Tree Star). For analysis of cytokines released by the alloreactive iT lymphocytes, we used a intracellular staining protocol (ebioscience) using anti-IL-17 (TC11-18H10.1, 1:100) and anti-IFN $\gamma$ (XMG1.2, 1:100) antibodies. For 
immunofluorescence staining, skin tissues were fixed in $4 \%$ buffered formalin (alfa aesar, $\mathrm{X} 07 \mathrm{~A} 031)$ at $4{ }^{\circ} \mathrm{C}$ overnight and cryosectioned $(5 \mu \mathrm{M})$. The infiltration of iT lymphocytes in the rejected skin tissues $(\mathrm{BALB} / \mathrm{c})$ were detected by immunofluorescence staining as described ${ }^{51}$. The primary anti-CD3 antibody (1:500, ab5690, Abcam) and secondary donkey anti-rabbit IgG conjugated with Alexa fluor-555 (1:1000, ab150062, abcam) were used. All slides were observed using a microscope (LSM800, Zeiss) and the images were processed by Adobe Photoshop Element software (version 4.0, Adobe Systems, San Jose, CA).

\section{CHIP-Seq and data analysis.}

One hundred and sixty million $\left(1.6 \times 10^{8}\right)$ enriched B220 ${ }^{+}$cells from ten Rosa26 $6^{\text {BirA/BirA }}$ transgenic mice were transduced with Bio-Hox 55 retro-virus (Bio-Hoxb5 pro-pre-B cells). The Bio-Hoxb5 pro-pre-B cells were cultured in vitro for three days prior to collection for streptavidin-mediated chromatin precipitation using a protocol described previously ${ }^{53},{ }^{54}$. Briefly, eight million sorted $\mathrm{GFP}^{+}$Bio-Hoxb5 pro-pre-B cells were fixed with $1 \%$ formaldehyde in culture medium for $12 \mathrm{~min}$ at room temperature followed by quenching with $0.125 \mathrm{M}$ glycine for $5 \mathrm{~min}$. The cells were washed twice with ice-cold PBS, and lysed in hypotonic buffer (20mM HEPES PH 7.5, 10mM KCl, 10\% Glycerol, 1mM EDTA, 0.2\% NP-40 plus protease inhibitor cocktail) for at least $30 \mathrm{~min}$ on ice to break the cell membrane. Then the cells were lysed in lysis buffer (1\% SDS, 10mM EDTA, 50mM Tris pH 8.0 plus protease inhibitor cocktail) for at least $30 \mathrm{~min}$ on $4^{\circ} \mathrm{C}$ to break the cell nucleus. The crosslinked chromatin were sonicated to an average fragment size of 300 bp by using a Bioruptor $^{\mathrm{TM}}$ (UCD-300TO) Sonicator. After preclearing with Protein G beads, one percent of the sonicated DNA fragments were used as CHIP-Seq input control, and the ninety-nine percent sonicated DNA fragments were incubated with streptavidin beads (Dynabeads Streptavidin M280) at $4^{\circ} \mathrm{C}$ overnight. Beads were washed with the following buffers: $2 \%$ SDS (twice); $0.1 \%$ deoxycholate, $1 \%$ Triton X-100, 2 mM EDTA, 50 mM HEPES, $500 \mathrm{mM}$ $\mathrm{NaCl}$ (once); $0.1 \%$ deoxycholate, $1 \%$ Triton X-100, 2 mM EDTA, $50 \mathrm{mM}$ HEPES, $150 \mathrm{mM}$ $\mathrm{NaCl}$ (triple); TE buffer (20 mM Tris-HCl, pH 8.0, 2 mM EDTA) (twice). To reverse crosslinks, 100ul elution buffer (50 mM Tris-HCl, $\mathrm{pH}$ 8.0, $10 \mathrm{mM}$ EDTA, 1\% SDS) was added to the pelleted beads and placed in $70^{\circ} \mathrm{C}$ water bath overnight. At the same time, the input chromatin sample was also added 100ul elution buffer for reverse cross-link along with pulldown samples. Proteinase $\mathrm{K}$ was added to the chromatin samples, followed incubation for 6 hours at $55^{\circ} \mathrm{C}$. Genomic DNA was isolated from the precipitated material by phenol extraction and ethanol precipitation. DNA segments from ChIP or sonication input control were end-repaired and ligated to indexed Illumina adaptors followed by low-cycle PCR (VAHTS ${ }^{\text {TM }}$ Universal DNA Library Prep Kit ND604). The resulting libraries were sequenced with a Illumina HiSeq X Ten platform. The sequencing raw data were converted to fastq files using illumina bcl2fastq software, and uploaded to Gene Expression Omnibus public database (GSE105057). The raw reads (Fastq files) were aligned to mouse genome (mm 10) using Bowtie2 package. Peak calling was performed using homer package and visualized using IGV software. 


\section{Statistical analysis.}

Kaplan-Meier survival analysis used Log-rank test. Statistical analysis of RNA-Seq data used the DESeq2, GSEA and GO packages. Other significant analyses were performed using unpaired student's $t$-test (GraphPad Prism, GraphPad Software Inc).

\section{Supplementary Material}

Refer to Web version on PubMed Central for supplementary material.

\section{ACKNOWLEDGMENTS}

This work was supported by grants from the Major National Research Project of China (Grant No. 2015CB964401, 2015CB964900), CAS Key Research Program of Frontier Sciences (QYZDB-SSW-SMC057), the Major Scientific and Technological Project of Guangdong Province (2014B020225005), the Strategic Priority Research Program of the Chinese Academic of Sciences (Grant No. XDA01020311), co-operation program from Guangdong Natural Science Foundation 2014A030312012 and the grants from the National Natural Science Foundation of China (Grant No 31471117, 81470281) to J.W., the National Key Research and Development Program of China (2017YFA0103401) to B.L. and (2017YFA0103402) to A. H., the Major National Research Project of China (2015CB964900) to J.D. and H.W. the National Natural Science Foundation of China (Grant No 31600948) to D.Y., the Major National Research Project of China (Grant No. 2015CB964404) and the National Natural Science Foundation of China (Grant No. 91642208) to Y-G.Y., National Natural Science Foundation of China (Grant No. 81770222), and National Institutes of Health (NIH) of USA (AI079087 and HL130724) to D.W. We also thank Dr. Tao Cheng (State Key Laboratory of Experimental Hematology, China), Dr. Duanqing Pei (CAS Key Laboratory of Regenerative Biology) and Dr. Emery H. Bresnick (University of Wisconsin-Madison) for comments on the manuscript. We are grateful to Dr. Zhihua Liu from Institute of Biophysics (CAS, China) for gifting us Rag $1^{-/-}$ mice. We also thank the animal center and instrument center of GIBH for the animal care, cell sorting, as well as skin imaging.

\section{References}

1. Heyworth C, Pearson S, May G \& Enver T Transcription factor-mediated lineage switching reveals plasticity in primary committed progenitor cells. EMBO J 21, 3770-3781, doi:10.1093/emboj/ cdf368 (2002). [PubMed: 12110589]

2. Kulessa H, Frampton J \& Graf T GATA-1 reprograms avian myelomonocytic cell lines into eosinophils, thromboblasts, and erythroblasts. Genes Dev 9, 1250-1262 (1995). [PubMed: 7758949]

3. Visvader JE, Elefanty AG, Strasser A \& Adams JM GATA-1 but not SCL induces megakaryocytic differentiation in an early myeloid line. EMBO J 11, 4557-4564 (1992). [PubMed: 1385117]

4. Xie H, Ye M, Feng R \& Graf T Stepwise reprogramming of B cells into macrophages. Cell 117, 663-676 (2004). [PubMed: 15163413]

5. Nutt SL, Heavey B, Rolink AG \& Busslinger M Commitment to the B-lymphoid lineage depends on the transcription factor Pax5. Nature 401, 556-562, doi:10.1038/44076 (1999). [PubMed: 10524622]

6. Rolink AG, Nutt SL, Melchers F \& Busslinger M Long-term in vivo reconstitution of T-cell development by Pax5-deficient B-cell progenitors. Nature 401, 603-606, doi:10.1038/44164 (1999). [PubMed: 10524629]

7. Taghon T, Yui MA \& Rothenberg EV Mast cell lineage diversion of T lineage precursors by the essential T cell transcription factor GATA-3. Nature immunology 8, 845-855, doi:10.1038/ni1486 (2007). [PubMed: 17603486]

8. Laiosa CV, Stadtfeld M, Xie H, de Andres-Aguayo L \& Graf T Reprogramming of committed T cell progenitors to macrophages and dendritic cells by C/EBP alpha and PU.1 transcription factors. Immunity 25, 731-744, doi:10.1016/j.immuni.2006.09.011 (2006). [PubMed: 17088084]

9. Li P et al. Reprogramming of T cells to natural killer-like cells upon Bcl11b deletion. Science 329, 85-89, doi:10.1126/science.1188063 (2010). [PubMed: 20538915] 
10. Cobaleda C, Jochum W \& Busslinger M Conversion of mature B cells into T cells by dedifferentiation to uncommitted progenitors. Nature 449, 473-477, doi:10.1038/nature06159 (2007). [PubMed: 17851532]

11. Ungerback J, Ahsberg J, Strid T, Somasundaram R \& Sigvardsson M Combined heterozygous loss of Ebf1 and Pax5 allows for T-lineage conversion of B cell progenitors. J Exp Med 212, 1109_ 1123, doi:10.1084/jem.20132100 (2015). [PubMed: 26056231]

12. Orkin SH \& Zon LI Hematopoiesis: an evolving paradigm for stem cell biology. Cell 132, 631644, doi:10.1016/j.cell.2008.01.025 (2008). [PubMed: 18295580]

13. Mansson R et al. Molecular evidence for hierarchical transcriptional lineage priming in fetal and adult stem cells and multipotent progenitors. Immunity 26, 407-419, doi:10.1016/j.immuni. 2007.02.013 (2007). [PubMed: 17433729]

14. Chen JY et al. Hoxb5 marks long-term haematopoietic stem cells and reveals a homogenous perivascular niche. Nature 530, 223-227, doi:10.1038/nature16943 (2016). [PubMed: 26863982]

15. Bijl J et al. Analysis of HSC activity and compensatory Hox gene expression profile in Hoxb cluster mutant fetal liver cells. Blood 108, 116-122, doi:10.1182/blood-2005-06-2245 (2006). [PubMed: 16339407]

16. Riddell $\mathrm{J}$ et al. Reprogramming committed murine blood cells to induced hematopoietic stem cells with defined factors. Cell 157, 549-564, doi:10.1016/j.cell.2014.04.006 (2014). [PubMed: 24766805]

17. Ramasamy I, Brisco M \& Morley A Improved PCR method for detecting monoclonal immunoglobulin heavy chain rearrangement in B cell neoplasms. J Clin Pathol 45, 770-775 (1992). [PubMed: 1401205]

18. Ehlich A, Martin V, Muller W \& Rajewsky K Analysis of the B-cell progenitor compartment at the level of single cells. Curr Biol 4, 573-583 (1994). [PubMed: 7953531]

19. Souabni A, Cobaleda C, Schebesta M \& Busslinger M Pax5 promotes B lymphopoiesis and blocks T cell development by repressing Notch1. Immunity 17, 781-793 (2002). [PubMed: 12479824]

20. Nechanitzky R et al. Transcription factor EBF1 is essential for the maintenance of B cell identity and prevention of alternative fates in committed cells. Nature immunology 14, 867-875, doi: 10.1038/ni.2641 (2013). [PubMed: 23812095]

21. Lin H \& Grosschedl R Failure of B-cell differentiation in mice lacking the transcription factor EBF. Nature 376, 263-267, doi:10.1038/376263a0 (1995). [PubMed: 7542362]

22. Liu P et al. Bcl11a is essential for normal lymphoid development. Nature immunology 4, 525-532, doi:10.1038/ni925 (2003). [PubMed: 12717432]

23. $\mathrm{Hu} \mathrm{H}$ et al. Foxp1 is an essential transcriptional regulator of B cell development. Nature immunology 7, 819-826, doi:10.1038/ni1358 (2006). [PubMed: 16819554]

24. Lin YC et al. A global network of transcription factors, involving E2A, EBF1 and Foxo1, that orchestrates B cell fate. Nature immunology 11, 635-643, doi:10.1038/ni.1891 (2010). [PubMed: 20543837]

25. Muller MR et al. Requirement for balanced Ca/NFAT signaling in hematopoietic and embryonic development. Proc Natl Acad Sci U S A 106, 7034-7039, doi:10.1073/pnas.0813296106 (2009). [PubMed: 19351896]

26. Braunstein M \& Anderson MK HEB in the spotlight: Transcriptional regulation of T-cell specification, commitment, and developmental plasticity. Clin Dev Immunol 2012, 678705, doi: 10.1155/2012/678705 (2012). [PubMed: 22577461]

27. Martins G \& Calame K Regulation and functions of Blimp-1 in T and B lymphocytes. Annu Rev Immunol 26, 133-169, doi:10.1146/annurev.immunol.26.021607.090241 (2008). [PubMed: 18370921]

28. Ferrando AA et al. Gene expression signatures define novel oncogenic pathways in T cell acute lymphoblastic leukemia. Cancer Cell 1, 75-87 (2002). [PubMed: 12086890]

29. Schwickert TA et al. Stage-specific control of early B cell development by the transcription factor Ikaros. Nature immunology 15, 283-293, doi:10.1038/ni.2828 (2014). [PubMed: 24509509]

30. Schebesta A et al. Transcription factor Pax 5 activates the chromatin of key genes involved in B cell signaling, adhesion, migration, and immune function. Immunity 27, 49-63, doi:10.1016/j.immuni. 2007.05.019 (2007). [PubMed: 17658281] 
31. McManus $\mathrm{S}$ et al. The transcription factor Pax5 regulates its target genes by recruiting chromatinmodifying proteins in committed B cells. EMBO J 30, 2388-2404, doi:10.1038/emboj.2011.140 (2011). [PubMed: 21552207]

32. McMahon KA et al. Mll has a critical role in fetal and adult hematopoietic stem cell self-renewal. Cell Stem Cell 1, 338-345, doi:10.1016/j.stem.2007.07.002 (2007). [PubMed: 18371367]

33. Artinger EL et al. An MLL-dependent network sustains hematopoiesis. Proc Natl Acad Sci U S A 110, 12000-12005, doi:10.1073/pnas.1301278110 (2013). [PubMed: 23744037]

34. Cobaleda C, Schebesta A, Delogu A \& Busslinger M Pax5: the guardian of B cell identity and function. Nature immunology 8, 463-470, doi:10.1038/ni1454 (2007). [PubMed: 17440452]

35. Vaillant F, Blyth K, Andrew L, Neil JC \& Cameron ER Enforced expression of Runx2 perturbs T cell development at a stage coincident with beta-selection. J Immunol 169, 2866-2874 (2002). [PubMed: 12218099]

36. Zhang H et al. MLL1 Inhibition Reprograms Epiblast Stem Cells to Naive Pluripotency. Cell Stem Cell 18, 481-494, doi:10.1016/j.stem.2016.02.004 (2016). [PubMed: 26996599]

37. Zhou X, Marks PA, Rifkind RA \& Richon VM Cloning and characterization of a histone deacetylase, HDAC9. Proc Natl Acad Sci U S A 98, 10572-10577, doi:10.1073/pnas.191375098 (2001). [PubMed: 11535832]

38. Krivega I, Dale RK \& Dean A Role of LDB1 in the transition from chromatin looping to transcription activation. Genes Dev 28, 1278-1290, doi:10.1101/gad.239749.114 (2014). [PubMed: 24874989]

39. Kokavec J et al. The ISWI ATPase Smarca5 (Snf2h) Is Required for Proliferation and Differentiation of Hematopoietic Stem and Progenitor Cells. Stem Cells 35, 1614-1623, doi: 10.1002/stem.2604 (2017). [PubMed: 28276606]

40. Starr TK, Jameson SC \& Hogquist KA Positive and negative selection of T cells. Annu Rev Immunol 21, 139-176 (2003). [PubMed: 12414722]

41. Limon A et al. High-titer retroviral vectors containing the enhanced green fluorescent protein gene for efficient expression in hematopoietic cells. Blood 90, 3316-3321 (1997). [PubMed: 9345013]

42. Goldschneider I, Komschlies KL \& Greiner DL Studies of thymocytopoiesis in rats and mice. I. Kinetics of appearance of thymocytes using a direct intrathymic adoptive transfer assay for thymocyte precursors. J Exp Med 163, 1-17 (1986). [PubMed: 3510267]

43. Wolfer A, Wilson A, Nemir M, MacDonald HR \& Radtke F Inactivation of Notch1 impairs VDJbeta rearrangement and allows pre-TCR-independent survival of early alpha beta Lineage Thymocytes. Immunity 16, 869-879 (2002). [PubMed: 12121668]

44. Ye J, Ma N, Madden TL \& Ostell JM IgBLAST: an immunoglobulin variable domain sequence analysis tool. Nucleic Acids Res 41, W34-40, doi:10.1093/nar/gkt382 (2013). [PubMed: 23671333]

45. Tang F et al. RNA-Seq analysis to capture the transcriptome landscape of a single cell. Nat Protoc 5, 516-535, doi: 10.1038/nprot.2009.236 (2010). [PubMed: 20203668]

46. Trapnell C et al. Differential gene and transcript expression analysis of RNA-seq experiments with TopHat and Cufflinks. Nat Protoc 7, 562-578, doi:10.1038/nprot.2012.016 (2012). [PubMed: 22383036]

47. Love MI, Huber W \& Anders S Moderated estimation of fold change and dispersion for RNA-seq data with DESeq2. Genome biology 15, 550, doi:10.1186/s13059-014-0550-8 (2014). [PubMed: 25516281]

48. Subramanian A et al. Gene set enrichment analysis: a knowledge-based approach for interpreting genome-wide expression profiles. Proc Natl Acad Sci U S A 102, 15545-15550, doi:10.1073/pnas. 0506580102 (2005). [PubMed: 16199517]

49. Yu G, Wang LG, Han Y \& He QY clusterProfiler: an R package for comparing biological themes among gene clusters. OMICS 16, 284-287, doi:10.1089/omi.2011.0118 (2012). [PubMed: 22455463]

50. Trickett A \& Kwan YL T cell stimulation and expansion using anti-CD3/CD28 beads. J Immunol Methods 275, 251-255 (2003). [PubMed: 12667688]

51. Lan P, Tonomura N, Shimizu A, Wang S \& Yang YG Reconstitution of a functional human immune system in immunodeficient mice through combined human fetal thymus/liver and CD34 ${ }^{+}$ 
cell transplantation. Blood 108, 487-492, doi:10.1182/blood-2005-11-4388 (2006). [PubMed: 16410443]

52. Jiang $\mathrm{X}$ et al. Skin infection generates non-migratory memory $\mathrm{CD} 8^{+} \mathrm{T}(\mathrm{RM})$ cells providing global skin immunity. Nature 483, 227-231, doi:10.1038/nature10851 (2012). [PubMed: 22388819]

53. Ai S et al. EED orchestration of heart maturation through interaction with HDACs is H3K27me3independent. Elife 6, doi:10.7554/eLife.24570 (2017).

54. He A et al. Dynamic GATA4 enhancers shape the chromatin landscape central to heart development and disease. Nat Commun 5, 4907, doi:10.1038/ncomms5907 (2014). [PubMed: 25249388] 


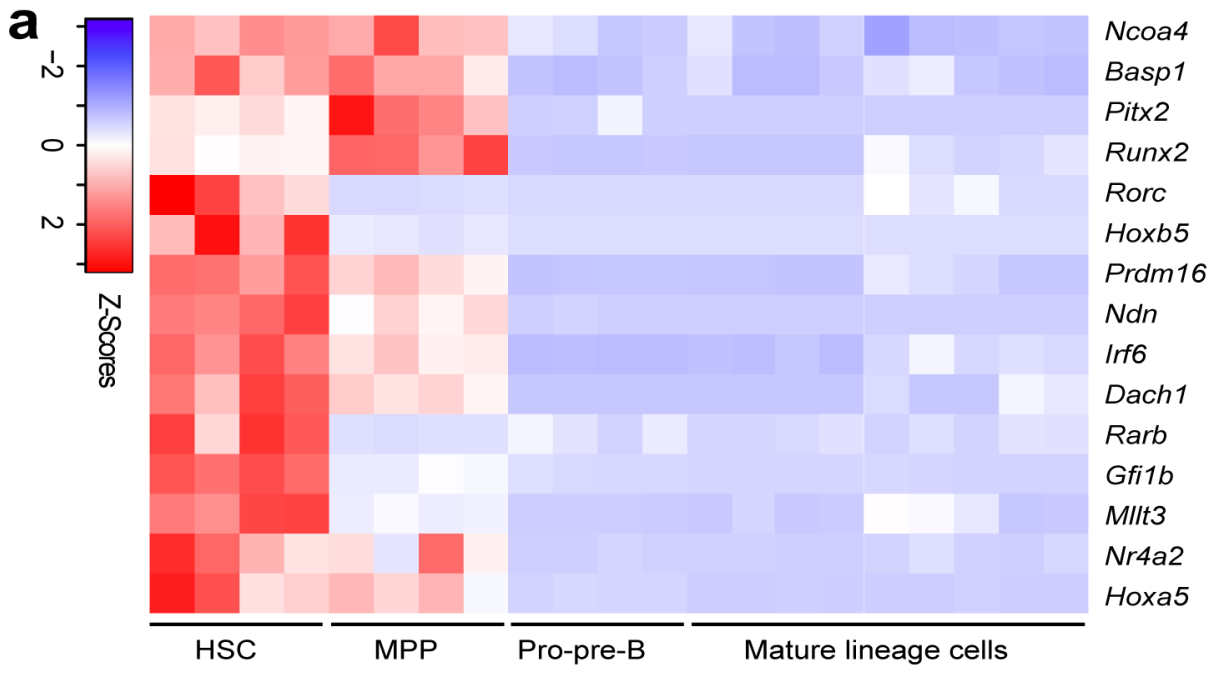

b

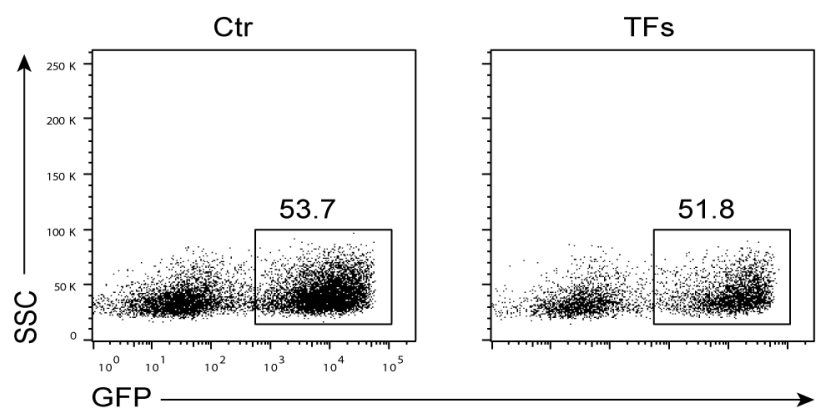

C

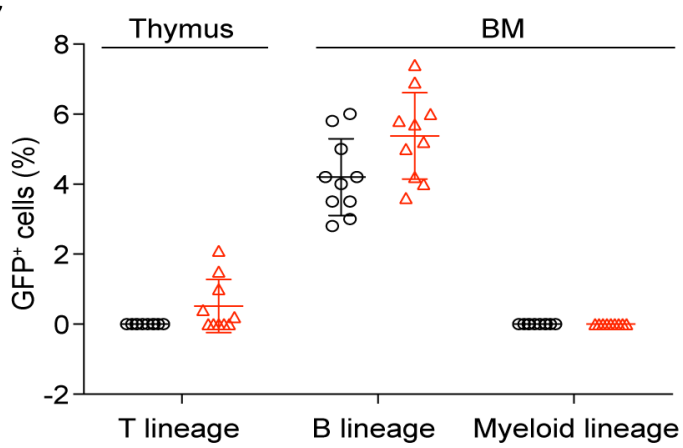

d

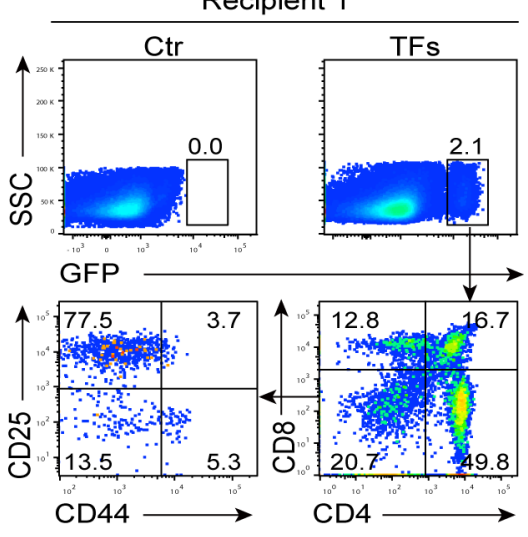

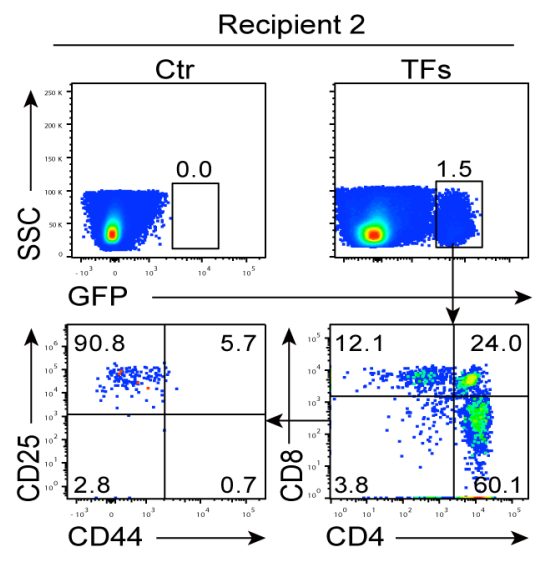

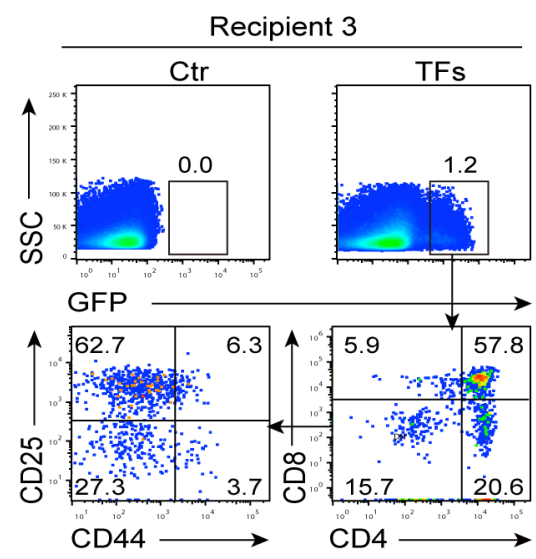

Figure 1. Screening for transcription factors involved in $\mathbf{B}$ to $\mathbf{T}$ cell conversion.

(a) Heatmaps of 15 transcription factors (TFs) preferentially-expressed in HSC and MPP, but not in pro-pre-B, mature T or B or myeloid cells. RNA-Seq was performed on 1000 cells of each cell type. HSC ( $n=4$ biologically independent samples), MPP ( $n=4$ biologically independent samples), pro-pre-B ( $\mathrm{n}=4$ biologically independent samples), mature lineage ( $n=9$ biologically independent samples). Genes for Heatmaps were screened by the principle of pairwise comparison (Significance: fold change $>2, \mathrm{P}<0.05$, two-sidedindependent Student's $t$ test). The fpkm values for each of 15 TFs were converted to z-score 
values (red, high; blue, low), and the heatmaps were plotted by gplots (heatmap.2). Columns represent the indicated biological replicates of each population. (b) Representative flow cytometry analysis of Ter119- $\mathrm{Mac1}^{-} \mathrm{CD} 3^{-} \mathrm{CD} 4^{-} \mathrm{CD} 8^{-} \mathrm{B} 220^{+} \mathrm{CD} 19^{+} \mathrm{CD} 93^{+} \mathrm{IgM}^{-}$pro-pre-B cells transduced with empty cassette or $15 \mathrm{TF}$ cocktail virus. Numbers above the gate indicate percent $\mathrm{GFP}^{+}$cells, (c) Percentage of $\mathrm{GFP}^{+}$cells in $\mathrm{T}$ lineage (thymus), B lineage and myeloid lineage (BM) of control-virus-transduced pro-pre-B cell recipients $(\mathrm{n}=10$ mice) and 15-TF-transduced pro-pre-B cell recipients ( $\mathrm{n}=10 \mathrm{mice}$ ) four weeks posttransplantation. Small horizontal lines indicate the mean ( \pm s.d.). (d) Flow cytometry analysis of $\mathrm{GFP}^{+}$lymphocytes in the thymus of 15-TF-transduced pro-pre-B cell recipient mice and control recipient mice four weeks post-transplantation $(n=3$ mice). Data are representative of four independent experiments (b) or are pooled from two independent experiments (c). 
a

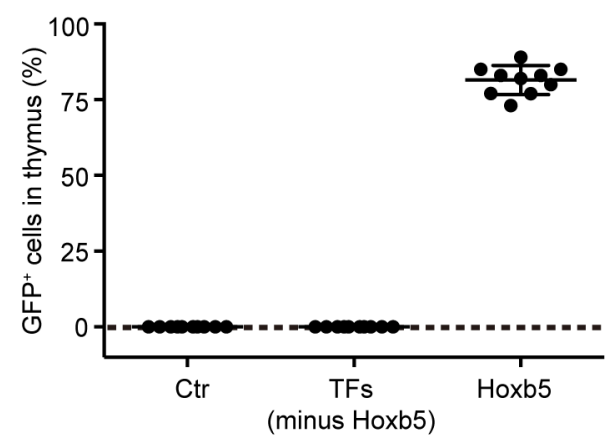

b

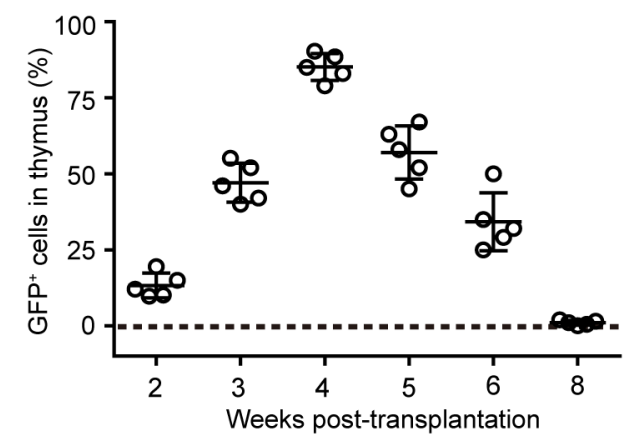

C
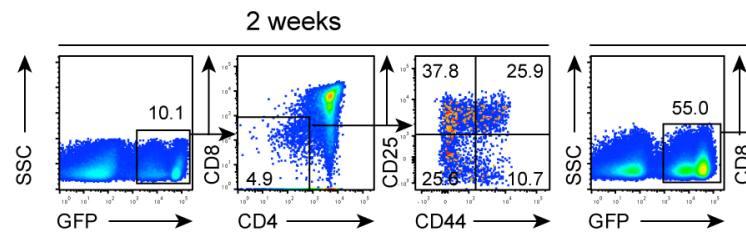

3 weeks
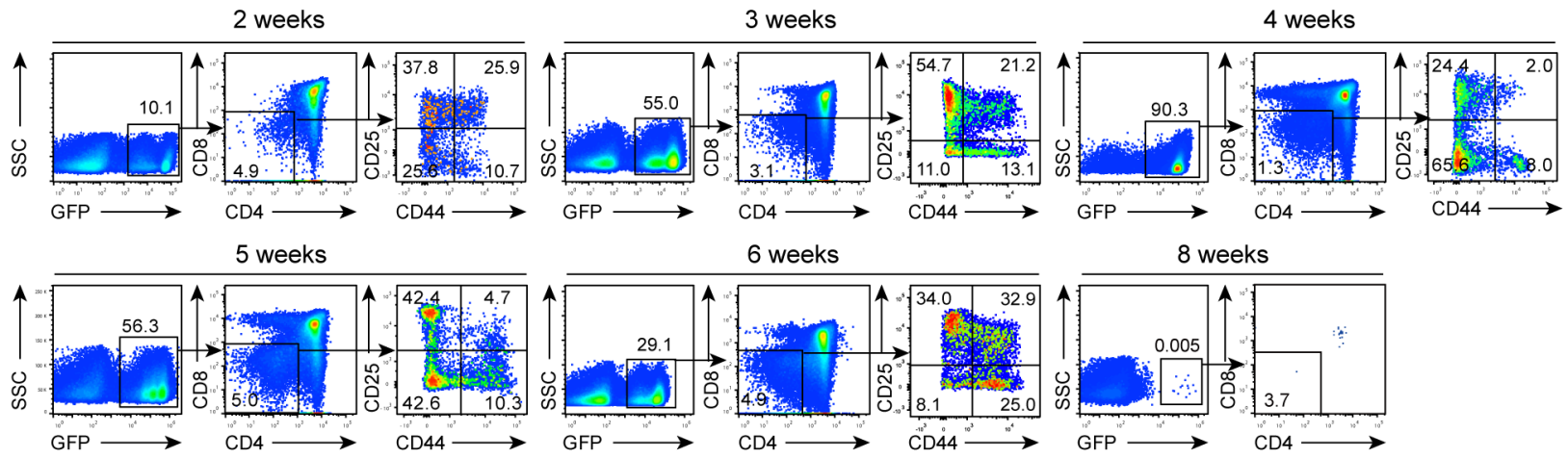

d
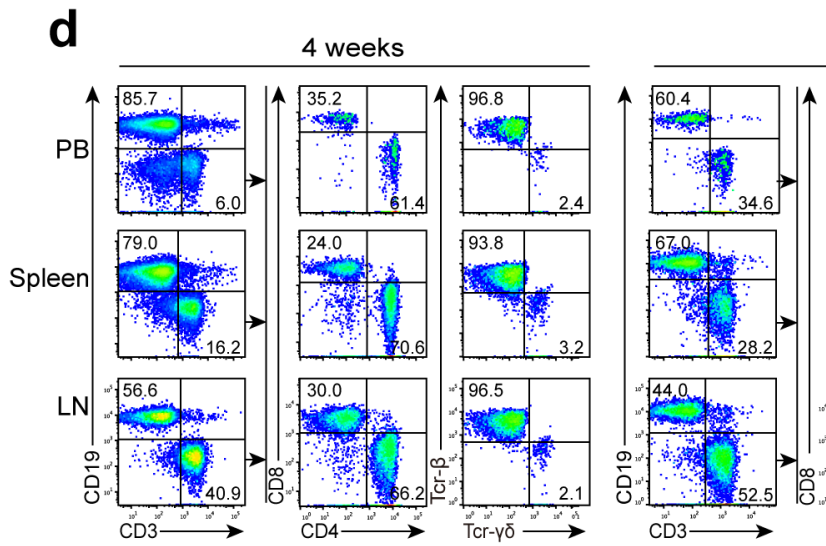

8 weeks
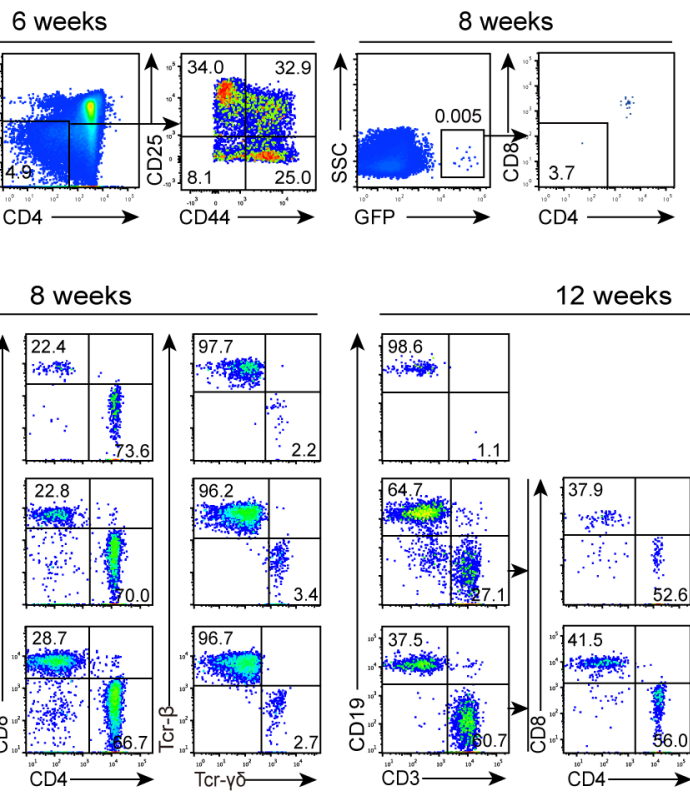

Figure 2. Expression of retro-Hoxb5 in pro-pre-B cells converts B cells to T lymphocytes in vivo. (a) Detection of Ter119- $\mathrm{Mac}^{-} \mathrm{CD} 19^{-} \mathrm{GFP}^{+}$cells in the thymus of recipients transplanted with $H o x b 5$ virus, GFP-control virus, or 14-factor virus cocktail lacking retro-Hoxb5 transduced pro-pre-B cells. Pro-pre-B cells transduced with the virus mentioned above were transplanted into sublethally irradiated individual congenic mice. Four weeks after transplantation, the Ter119- $\mathrm{Mac}^{-} \mathrm{CD} 19^{-} \mathrm{GFP}^{+}$cells in thymus were analysed by flow cytometry $(\mathrm{n}=10$ mice). Each symbol represents an individual mouse, and small horizontal lines indicate the mean ( \pm s.d.). (b) Kinetics of appearance of GFP ${ }^{+}$thymic cells in Hoxb5 recipient mice ( $\mathrm{n}=5$ mice). Each symbol represents an individual mouse, and small horizontal lines indicate the mean ( \pm s.d.). (c) Representative flow cytometry data showing kinetics of appearance of $\mathrm{GFP}^{+}$cells in the thymus of retro-Hoxb5 mice 2, 3, 4, 5, 6, and 8 
weeks post-transplantation. (d) Dynamics of $\mathrm{GFP}^{+} \mathrm{T}$ lymphocytes in PB, Spleen, and LN gated from $\mathrm{GFP}^{+} \mathrm{Ter} 11^{-} \mathrm{Mac1}^{-}$population of retro-Hoxb5 mice. Representative data from 4, 8 and 12 weeks post-transplantation were shown. Data are pooled from three independent experiments (a) or are representative of two independent experiments $(\mathbf{c}, \mathbf{d})$. 
a
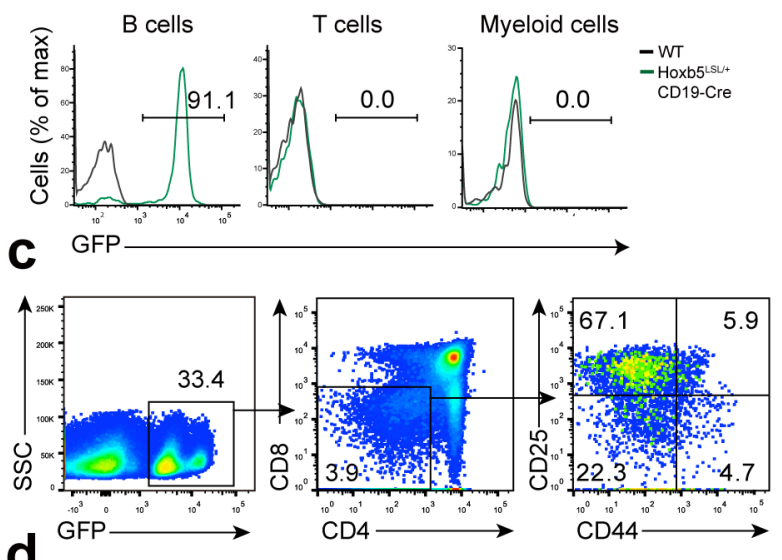

d

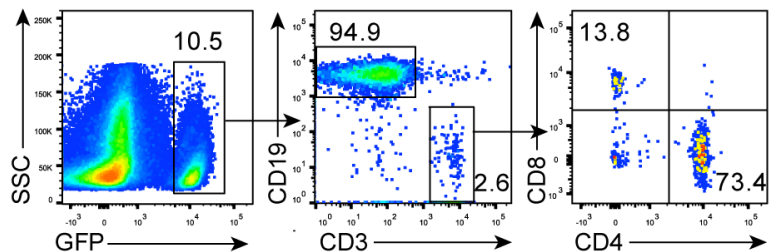

b

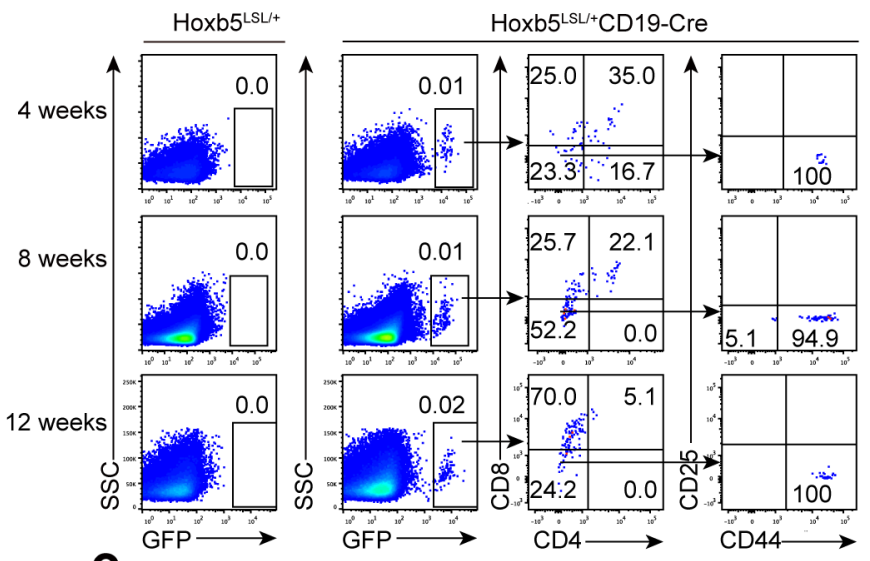

e

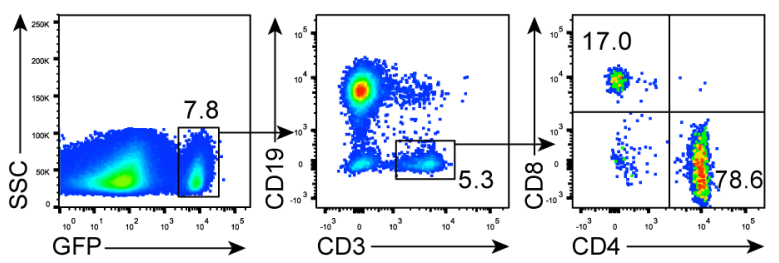

Figure 3. Conversion of B to T lymphocytes using a CD19-Hoxb5 transgenic model.

(a) GFP reported B-lineage specific expression of ectopic Hoxb5 in the PB of Hoxb5 $5^{\mathrm{LS} /+}$ CD19-Cre compound (CD19-Hoxb5) mice. CD19 ${ }^{+} \mathrm{B}$ cells, CD3 ${ }^{+} \mathrm{T}$ cells, and $\mathrm{Mac}^{+}$ Myeloid cells were analyzed by flow cytometry. Numbers above bracketed lines indicate percent $\mathrm{GFP}^{+}$cells, (b) Flow cytometry analysis of thymic Ter119- $\mathrm{Mac}^{-} \mathrm{CD}^{-} 9^{-} \mathrm{GFP}^{+}$ lymphocytes of 4, 8, and 12 weeks old CD19-Hoxb5 mice and littermate control mice under homeostasis. (c-e) Flow cytometry analysis of iDN cells in the thymus gated from Ter119- Mac1 $^{-}$CD19- ${ }^{-}$population (c), iT cells in spleen (d), and LN (e) gated from Ter119- $\mathrm{Mac1}^{-}$population of a representative recipient transplanted with three million CD19-Hoxb5 pro-pre-B cells four weeks after transplantation. Data are representative of two independent experiments $(\mathbf{a}, \mathbf{b})$ or three independent experiments $(\mathbf{c}, \mathbf{d}$ and $\mathbf{e})$. 
a

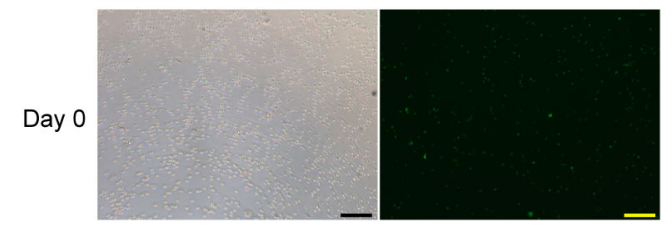

Day 6

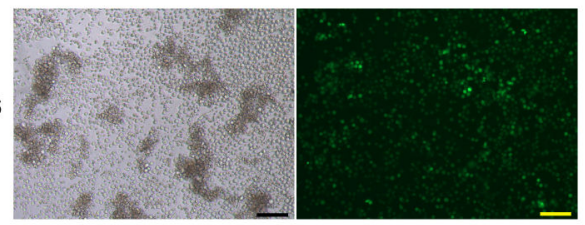

C
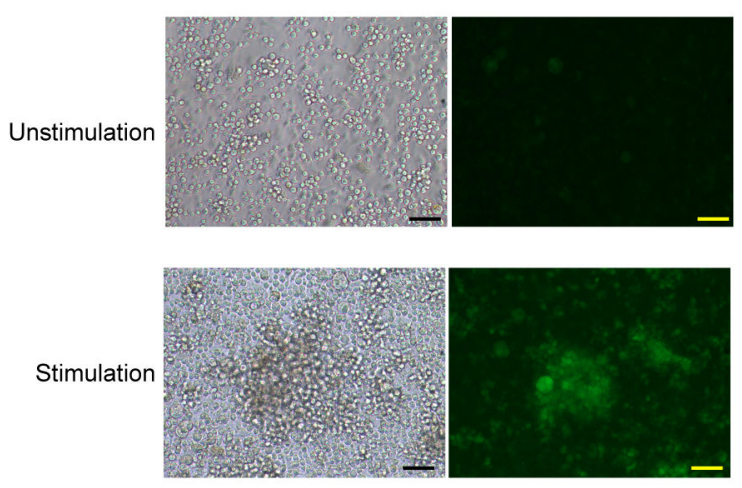

b

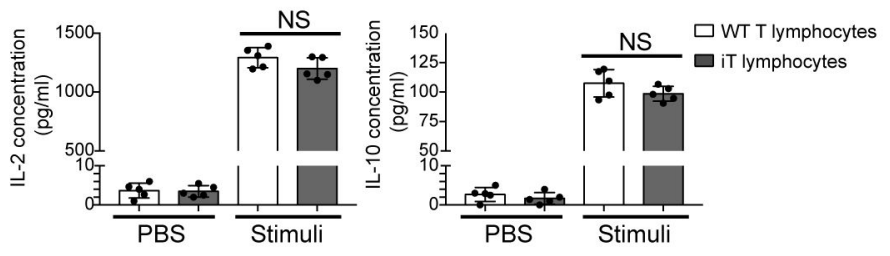

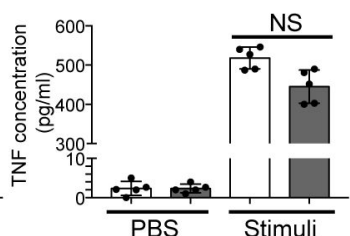

d

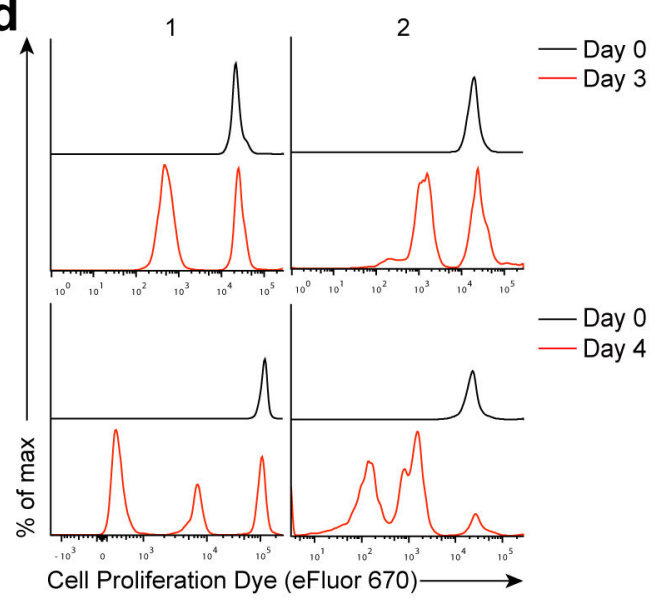

Figure 4. Immune functions of $\boldsymbol{H o x b 5}$-induced iT lymphocytes.

(a) Morphology of iT proliferation assay stimulated by anti-CD3 and anti-CD28 mAbs. Scale bar, $100 \mu \mathrm{M}$. (b) ELISA analysis of IL-2, IL-10, IFN- $\gamma$ and TNF secreted by splenic iT lymphocytes after stimulation in vitro. Splenic iT lymphocytes were sorted ( 0.2 million/ well, 96-well plate) and stimulated by anti-CD3 mAb (50 $\mu \mathrm{g} / \mathrm{ml})$ and anti-CD28 mAb (5 $\mu \mathrm{g} / \mathrm{ml}$ ) for 6 days. Each symbol represents an individual sample, and small horizontal lines indicate the mean ( \pm s.d.). (NS, not significant $(\mathrm{P}>0.05$ ) two-sided-independent $t$-test, $\mathrm{n}=5$ biologically independent samples). (c) Morphology of iT blasts stimulated by inactivated allogeneic splenocytes (BALB/c mouse) on day 7. Scale bar, $100 \mu \mathrm{m}$. (d) Flow cytometry analysis of proliferative $\mathrm{CD} 4^{+}$iT lymphocytes in mixed lymphocyte reaction assay. Splenic iT lymphocytes from representative Hoxb5-Rag $1^{-/ \text {" }}$ mice with (1)/without (2) allogeneic skin transplantation were analysed. Half million sorted splenic iT lymphocytes were stained by cell proliferation dye eFluor 670 , then co-cultured with 0.5 million inactivated allogeneic splenocytes (BALB/C) in individual wells (96 well plates). After three to four days, the cells were collected and analysed by flow cytometry. Data are representative of three independent experiments $(\mathbf{a}, \mathbf{c}, \mathbf{d})$ or are pooled from two independent experiments (b). 
a

C CD45.2 ${ }^{+}$Ter119 ${ }^{-} \mathrm{Mac}^{-}{ }^{-} \mathrm{CD} 19^{-} \mathrm{CD} 4{ }^{+} \mathrm{CD} 44^{\mathrm{hi}} \mathrm{CD} 69^{+}$or

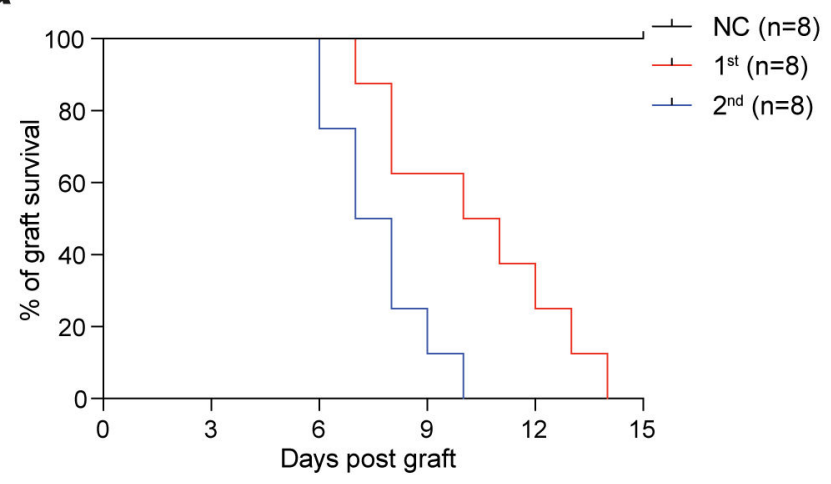

b
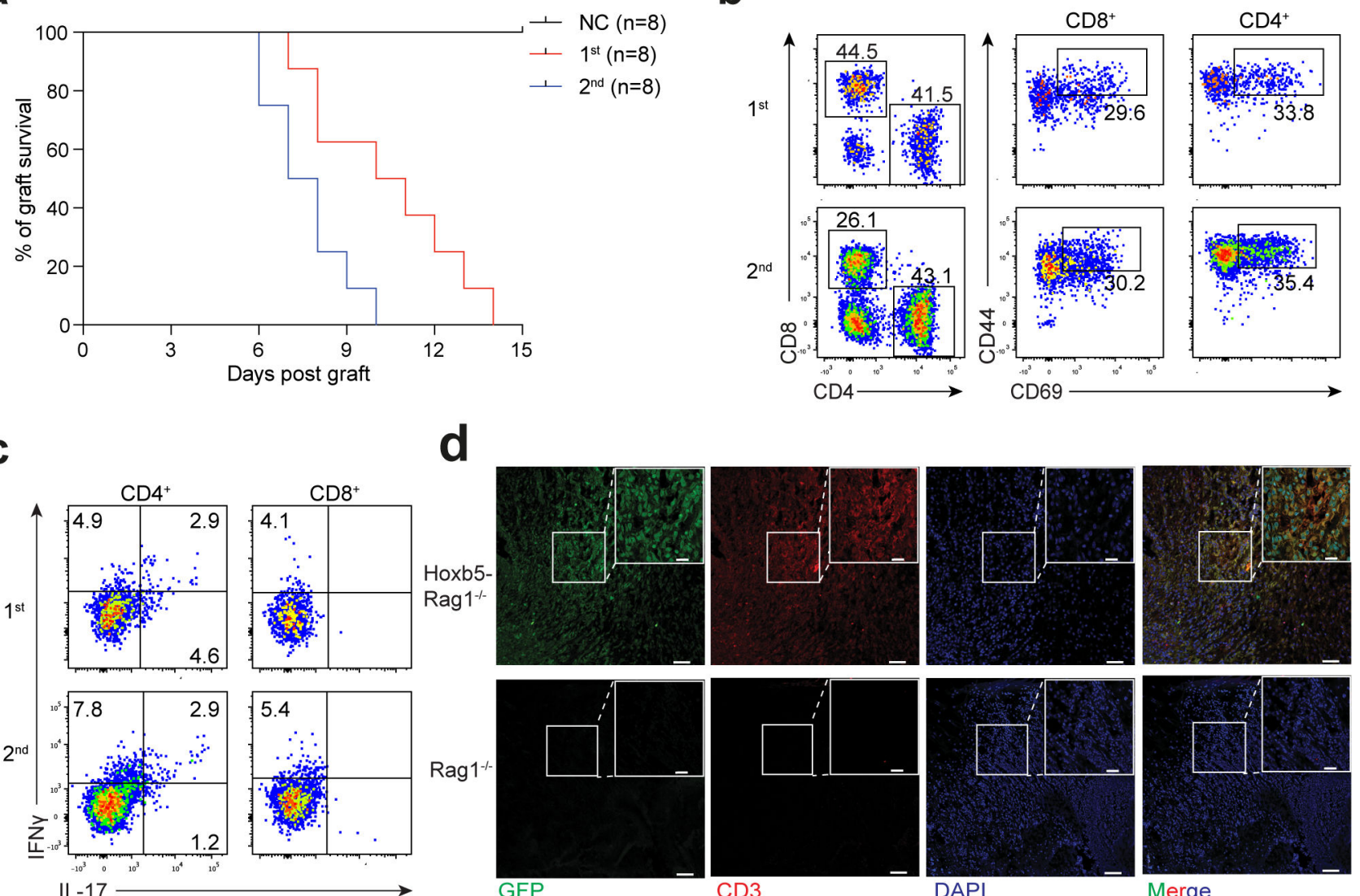

Merge

Figure 5. Hoxb5-induced iT lymphocytes show normal immune functions in vivo.

(a) Kaplan-Meier survival curves of allogeneic skin grafts from Rag1-/- (NC group, $\mathrm{n}=8$ mice) and Hoxb5-Rag1 $1^{-/-}$mice ( $\mathrm{n}=8$ mice). Median allogeneic graft survival: Primary allograft from Hoxb5-Rag $1^{-/-}=10.5 \mathrm{~d}$ ( $1^{\text {st }}$ group, $\mathrm{n}=8$ mice $)$; Secondary allograft from Hoxb5-Rag $1^{-/-}=7.5 \mathrm{~d}$ ( $2^{\text {nd }}$ group, $\mathrm{n}=8$ mice) ( $\mathrm{P}$ value $<0.001$, Log-rank test). Time interval between primary and secondary allogeneic skin graft $=8$ weeks. (b) Flow cytometry analysis of activation status of Hoxb5-induced iT lymphocytes in the allogeneic skin graft. Representative plots from rejected primary allogeneic skin grafts $\left(1^{\text {st }}\right.$, day 10$)$ and rejected secondary allogeneic skin grafts $\left(2^{\text {nd }}\right.$, day 6$)$ were shown. Single cells were harvested from rejected skin tissues by collagenase I digestion. Activated iT cells were defined as CD45.2 ${ }^{+}$Ter1 19- $\mathrm{Mac}^{-}{ }^{-} \mathrm{CD} 19^{-} \mathrm{CD}^{+} \mathrm{CD} 44^{\mathrm{hi}} \mathrm{CD} 69^{+}$. (c) Intra-cellular staining of IFN $\mu$ and IL-17 in the activated CD45.2 ${ }^{+} \mathrm{CD}^{+}$or $\mathrm{CD}^{+}$iT lymphocytes from the allogeneic skin grafts. Representative plots from rejected primary allogeneic skin grafts ( $1^{\text {st }}$, day 10$)$ and rejected secondary allogeneic skin grafts $\left(2^{\text {nd }}\right.$, day 6) were shown. (d) Immunofluorescence staining of $\mathrm{GFP}^{+} \mathrm{CD}^{+}$iT lymphocytes in allogeneic skin grafts. Images from representative allogeneic skin tissue sections from Rag1 ${ }^{-/-}$and Hoxb5-Rag1 ${ }^{-/-}$mice were shown. Scale bars, $20 \mu \mathrm{m}$ or $50 \mu \mathrm{m}$. Data are representative of three independent experiments (b-d). 

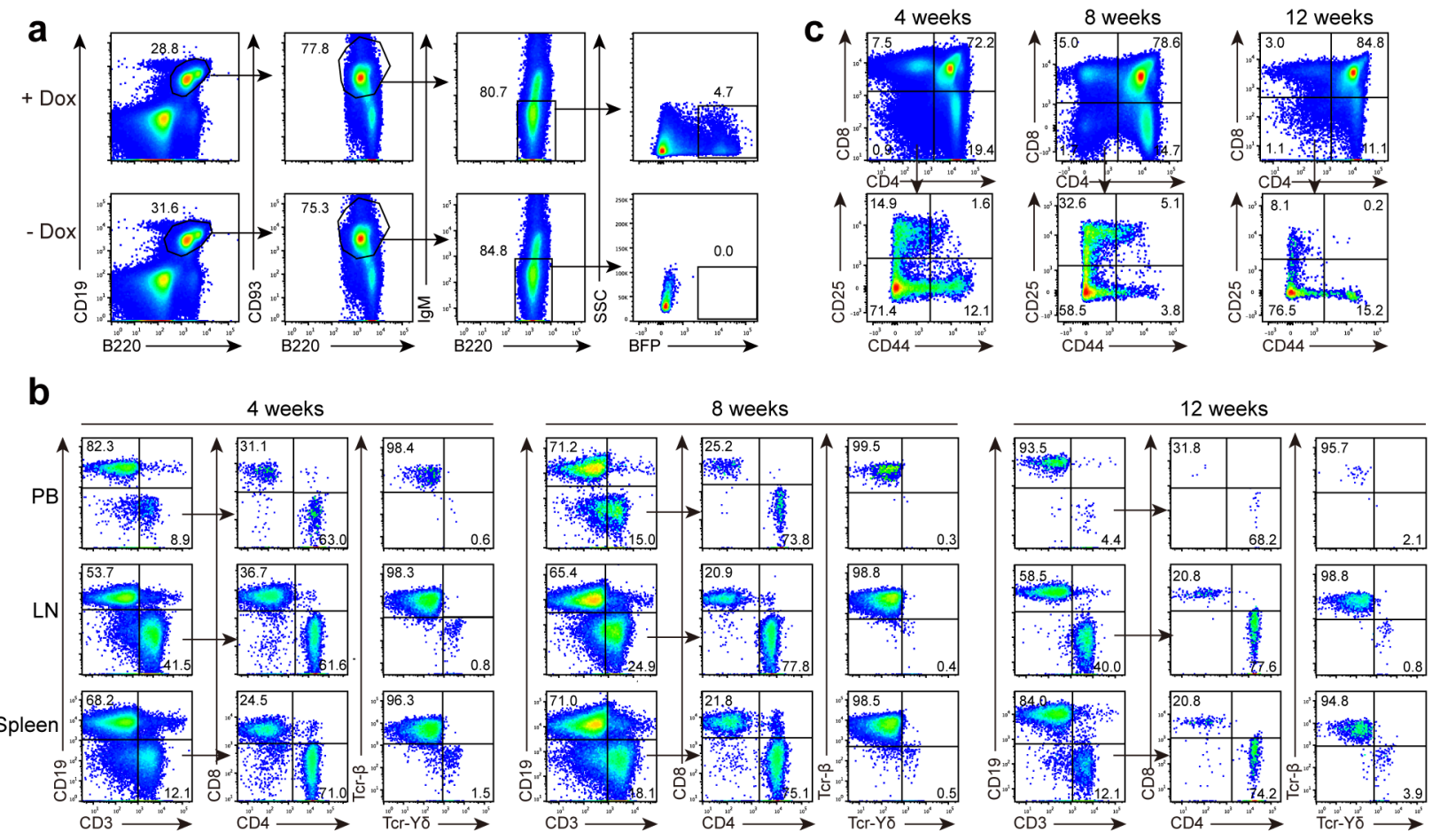

Figure 6. Transient expression of Hoxb5 reprograms B cells into T lymphocytes using a TetHoxb5 transgenic model.

(a) Flow cytometry analysis of $\mathrm{BFP}^{+} \mathrm{B} 220^{+} \mathrm{CD} 19^{+} \mathrm{CD}_{3}{ }^{+} \mathrm{IgM}^{-}$pro-pre-B cells in the bone marrow of a representative Tet-Hox 55 mouse, which was maintained on drinking water with doxycycline $(1 \mathrm{mg} / \mathrm{ml})$ for one week. One Tet-Hoxb5 mouse without feeding Dox water was used as negative control. (b-c) Dynamics of iT lymphocytes in PB, Spleen, and LN (b) gated from $\mathrm{CD} 45.2^{+}$Ter $119^{-} \mathrm{Mac1}^{-}$population, and iDN cells in thymus (c) gated from CD45.2 $2^{+}$Ter119- Mac1 $^{-} \mathrm{CD} 19^{-}$population of recipients transplanted with Tet-Hoxb5 propre-B cells. Representative mice were analysed 4 weeks, 8 weeks and 12 weeks after transplantation. Data are representative of two independent experiments $(\mathbf{b}, \mathbf{c})$. 


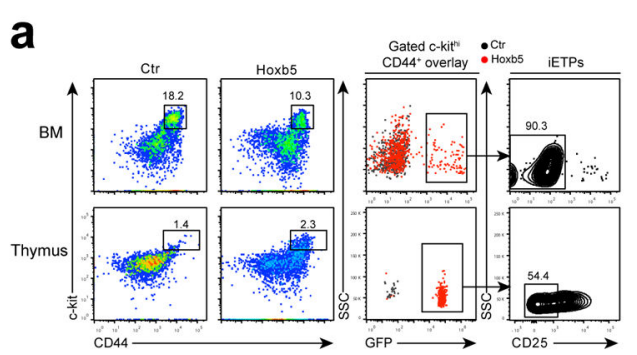

b

C

d

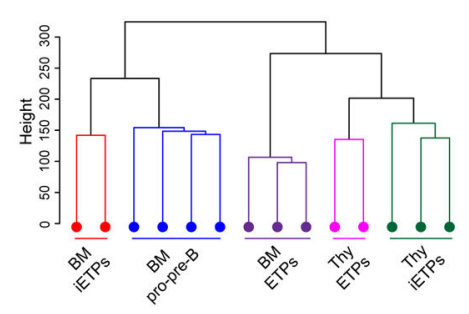

e
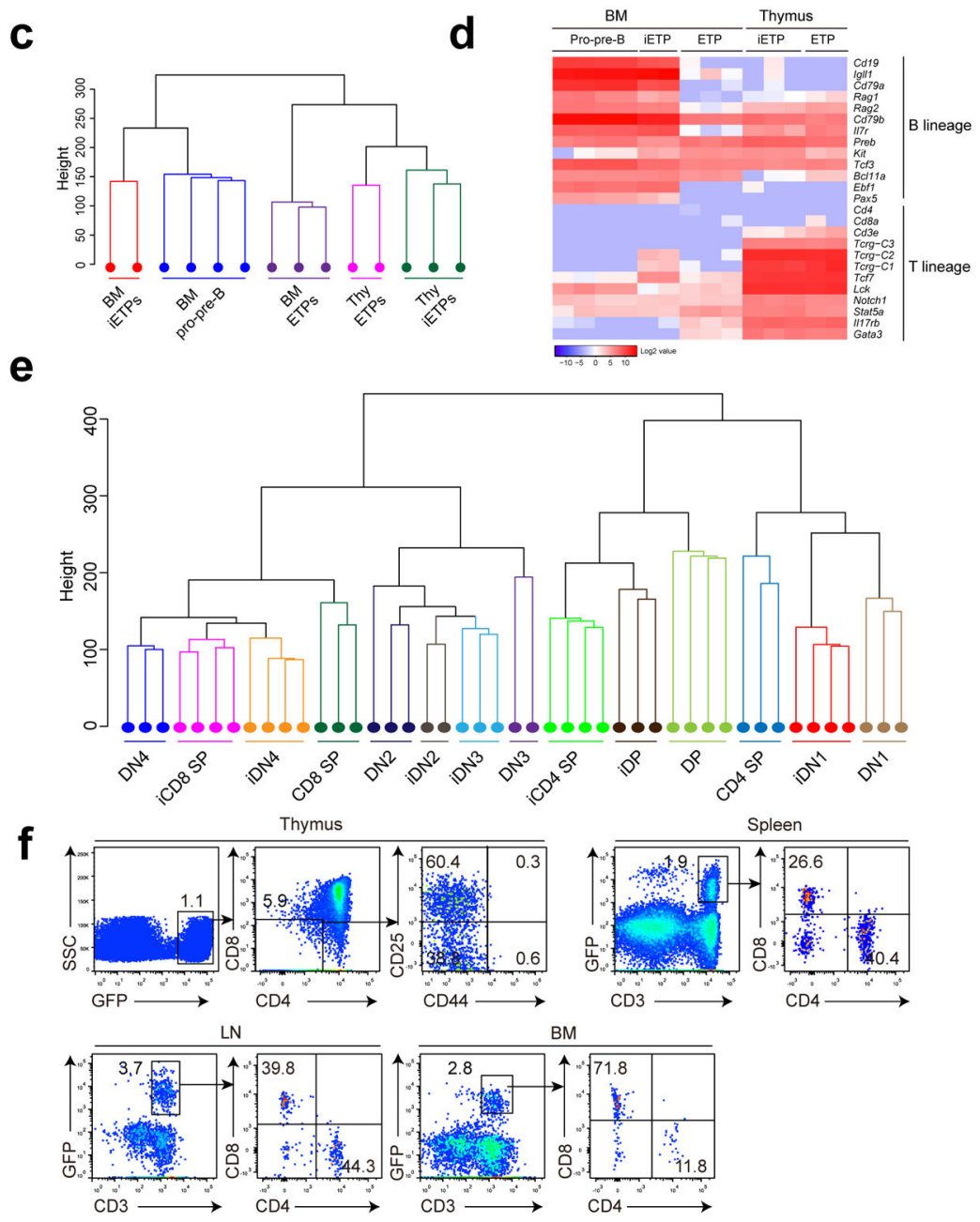

Figure 7. Hoxb5 directly converts B lymphocytes to early $T$ cell progenitor-like cells in bone marrow.

(a) Flow cytometry analysis of iETPs in the BM and thymus of a representative retro- $\mathrm{Hox} b 5$ recipient four weeks post-transplantation. Five million sorted retro-Hox 65 pro-pre-B cells were transplanted into sublethally irradiated individual recipients. Recipients transplanted with pro-pre-B cells transduced with GFP control virus were used as controls.

Representative plots from five mice of each groups were shown. (b) Absolute numbers of iETP cells in the BM and thymus of $H o x b 5$ recipients ( $\mathrm{n}=5$ mice) at different time points after transplantation. Each symbol represents an individual mouse, and small horizontal lines indicate the mean ( \pm s.d.). (c) Unsupervised hierarchical clustering of RNA-Seq data of 
iETPs, natural ETPs and pro-pre-B cells. For each RNA-Seq sample, one thousand iETPs or ETP $\left(\mathrm{Lin}^{-} \mathrm{CD} 44^{+} \mathrm{c}-\mathrm{kit}^{\mathrm{hi}} \mathrm{CD} 25^{-}\right.$) from retro-Hoxb5 mice or wild type control mice were sorted and analysed. (d) Heatmaps showed the expression pattern of selected genes related to T or B cells between iETPs, natural ETPs and pro-pre-B cells. Columns represent the indicated cell subsets in biological replicates. (e) Unsupervised hierarchical clustering of RNASeq data of iDN1, iDN2, iDN3, iDN4, iDP, iCD4-SP, iCD8-SP cells and related wild type counterparts. (f) Flow cytometry analysis of iT lymphocytes in thymus, spleen, LN and $\mathrm{BM}$ of a representative recipient 3 weeks after secondary transplantation of iT thymocytes. Data are representative of two independent experiments. 
a

b
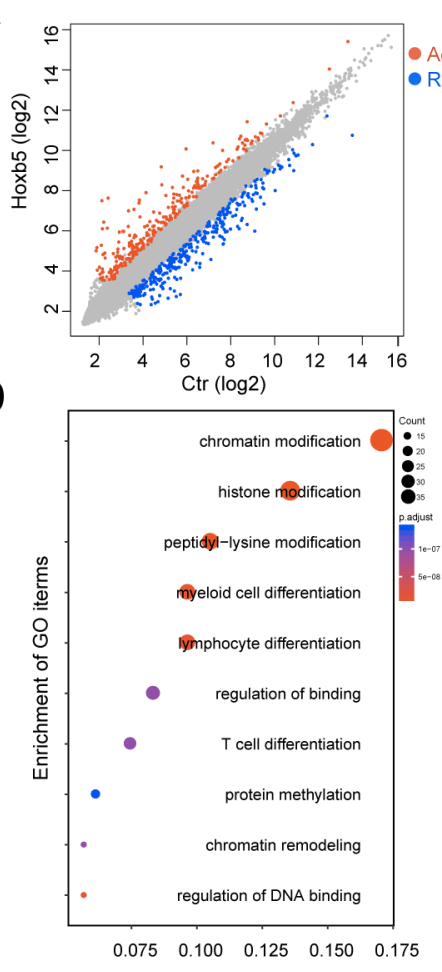

C

- Activated - Repressed

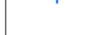

h
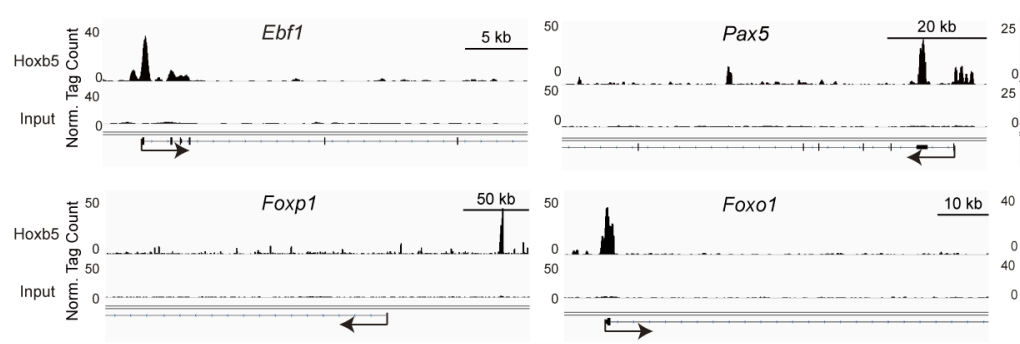

$10 \mathrm{~kb} \quad 40$

d

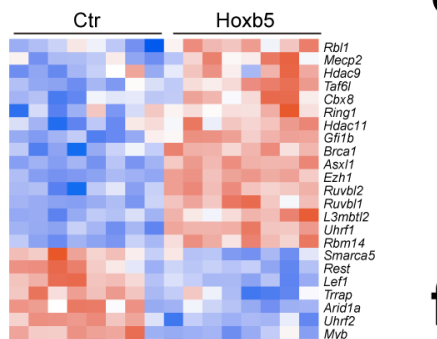

$f^{\alpha}$
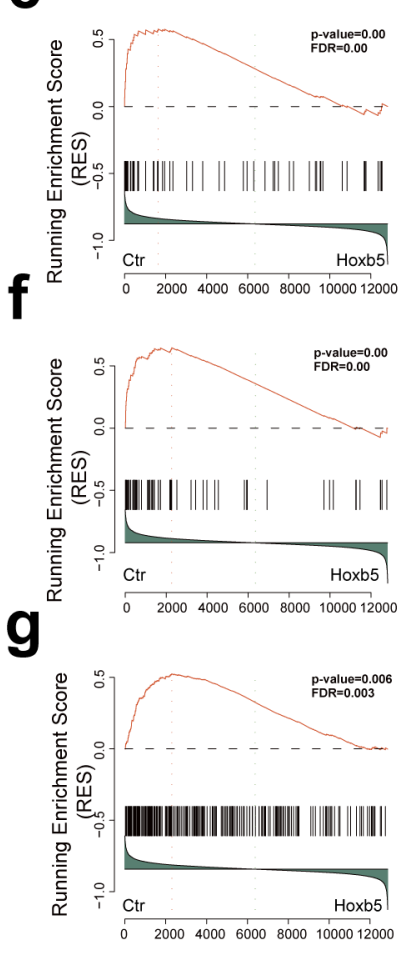

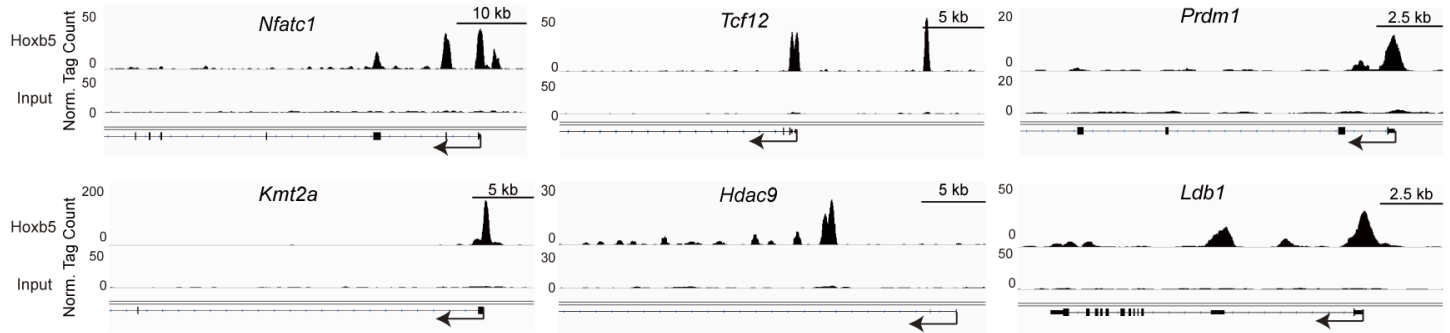

Figure 8. Hoxb5 targets in pro-pre-B cells.

(a) Scatter plot of gene expression differences between empty vector transduced pro-pre-B cells and retro-Hoxb5 pro-pre-B cells. The normalized expression value (mean) of each gene in the two groups was plotted as fragments per kilobase million (fpkm). Differential genes significantly up-regulated (red) and down-regulated (blue) in retro-Hox 65 pro-pre-B cells were highlighted ( $\mathrm{n}=8$ biologically independent samples, fold $>2, \mathrm{p}$ adj $<0.05$, DESeq2 $\mathrm{R}$ packages). (b) Gene ontology (GO) enrichment analysis of the 232 differentially expressed transcription factors using clusterProfiler R packages. Each GO term is represented by a 
single circle, that the color indicates the p-values and the significant level of the GO term, and the size of each circle is proportional to the gene numbers. (c) Heatmaps of chromatin modification related transcription factors selected from the DEG list. The fpkm values of the chromatin modification related transcription factors were converted to z-score values (red, high; blue, low), and the heatmaps were plotted by gplots (heatmap.2). Columns represent the indicated cell subsets in eight biological replicates. (d) Heatmaps of lymphopoiesis related transcription factors from the DEG list. Columns represent the indicated cell populations in eight biological replicates. Gene set enrichment analysis showed that the Ikzf1 (Ikaros) activated targets (e), the Pax 5 activated targets (f), and the Kmt2a (MII) activated targets in HPC (CD48 ${ }^{-}$LSK) (g) were repressed in retro- ${ }^{-H o x b 5}$ pro-pre-B cells. (h) CHIP-Seq profiles Hoxb5 binding tracks in pro-pre-B on Ebf1, Pax5, Bcl11a, Foxp1, Foxo1, Lmo2, Nfatc1, Tcf12, Prdm1, Kmt2a (MII), Hdac9, and Ldb1 gene locus. Data are representative of two independent experiments. 